\title{
Virtual Scanning Algorithm for Road Network Surveillance
}

\author{
Jaehoon Jeong, Student Member, IEEE, Yu Gu, Student Member, IEEE, Tian He, Member, IEEE, \\ and David H.C. Du, Fellow, IEEE
}

\begin{abstract}
This paper proposes a $\underline{\text { VIrtual }} \underline{\text { Scanning }} \underline{\text { Algorithm }}$ (VISA), tailored and optimized for road network surveillance. Our design uniquely leverages upon the facts that (i) the movement of targets (e.g., vehicles) is confined within roadways and (ii) the road network maps are normally known. We guarantee the detection of moving targets before they reach designated protection points (such as temporary base camps), while maximizing the lifetime of the sensor network. The main idea of this work is virtual scan - waves of sensing activities scheduled for road network protection. We provide design-space analysis on the performance of virtual scan in terms of lifetime and average detection delay. Importantly, to our knowledge, this is the first work to study how to guarantee target detection while sensor network deteriorates, using a novel hole handling technique. Through theoretical analysis and extensive simulation, it is shown that a surveillance system, using our design, sustains orders-of-magnitude longer lifetime than full coverage algorithms, and as much as ten times longer than legacy duty cycling algorithms.
\end{abstract}

Index Terms-Sensor Network, Road Network, Virtual Scanning, Surveillance, Detection, Protection.

\section{INTRODUCTION}

Surveillance for critical infrastructure and areas is regarded as one of the most practical applications of wireless sensor networks (WSNs). So far, most of WSN surveillance systems have focused on surveillance for two-dimensional spaces, such as open battlefields [1]-[4]. Research on road network surveillance, however, is very limited. In modern warfare, roadways (as fast maneuver paths) are vantage areas for military surveillance and operations. Clearly, surveillance in a road network is significantly different, because (i) the movement of targets (e.g., vehicles) is confined within road segments, and (ii) the road network maps are normally known (e.g., from Google Earth and Yahoo Maps). We argue that legacy solutions, which are not tailored for road networks, lead to suboptimal performance.

This paper proposes a novel sensing scheduling algorithm for target intrusion detection, utilizing the unique features of road networks. Specifically, we focus on supporting military operations with fast, infrastructure-free deployment. As shown in Figure 1(a), we guarantee the detection of targets, entering from entrance points, before they reach one of protection points; in modern

- Jaehoon Jeong, Yu Gu, Tian He, and David Du are with the Department of Computer Science and Engineering, University of Minnesota, Twin Cities, 200 Union Street SE, Minneapolis, MN 55455.

E-mail: \{jjeong,yugu,tianhe,du\}@cs.umn.edu.

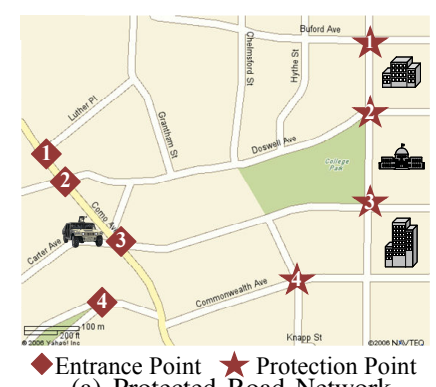

(a) Protected Road Network

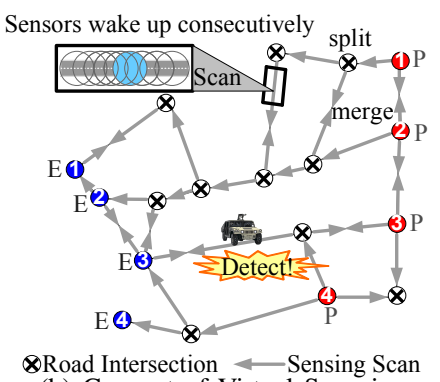

(b) Concept of Virtual Scanning

\section{Fig. 1. Road Network Surveillance}

warfare, battlefield situational awareness requires both entrance points and protections points (e.g., temporary base camps) to be assigned and changed on demand for fast military maneuver within a road network. Therefore, we cannot place sensor gates a priori before protection points for intrusion detection. Instead, a roadnetwork-wide deployment is needed.

A straightforward solution for road network surveillance is duty cycling, in which nodes wake up simultaneously for $w$ seconds (the minimum working time before reliable detection can be reported) and then the whole network remains silent for $T$ seconds. The detection is guaranteed if it takes more than $T$ seconds for a target to travel along the shortest path between any pair of entrance points and protection points; this duty-cycling-based algorithm performs much better in terms of system lifetime than traditional full coverage algorithms [1]-[4] in road networks. This is because the duty cycling algorithm allows the whole network to be silent completely for $T$ seconds every $w$ seconds, but the full coverage algorithms (e.g., the one covers all intersections) require at least one subset of sensors to be active at any given point time, taking no advantage of the linear structure of road networks.

In this paper, we present a novel scan-based algorithm, which improves further energy efficiency of surveillance in road networks. As shown in Figure 1(b), sensors wake up one by one for $w$ seconds along road segments, creating waves of sensing activities, called virtual scanning. Waves propagate from one (or multiple) protection point $P$, split at the intersections, and merge along the route until they scan all of the road segments under surveillance. Our study reveals that this scan-based method can achieve significantly better performance (e.g., ten times system lifetime) than duty cycling algorithms. The concept of virtual scanning is simple, however, in-depth design is very challenging due to 
a set of practical issues we consider in this paper. Particularly, we investigate (i) how to optimize the network-wide silent duration $T$ between scan waves, (ii) how to coordinate the working schedules of individual sensors during the scan, and (iii) how to deal with sensing holes due to unbalanced initial node deployment, node failure and the depletion of node energy over time. Specifically, the intellectual contributions in this paper are as follows:

- A new architecture for surveillance in road networks. VISA is the first work tailored for road networks, leading to ordersof-magnitude longer system life for target intrusion detection, using a novel scan-based algorithm.

- A sensing scheduling algorithm for an arbitrary road network. The working schedule of each sensor (i.e., when to wake up) is constructed in a decentralized way. The network-wide silent duration is computed by VISA scheduler and naturally disseminated along with sensing waves to the nodes in a network.

- An optimal sensing hole handling algorithm for uncovered road segments. The VISA scheduler deals with both the initial sensing holes at the deployment time as well as the sensing holes due to the heterogeneous energy budget among sensors by optimally labeling additional pseudo protection or entrance points.

- Considerations on two practical issues: (i) Detection failure probability and (ii) Time synchronization error. For each issue, we propose an optimal solution in terms of network lifetime.

The rest of this paper is organized as follows: Section 2 describes the problem formulation. Section 3 explains the VISA system design. Section 4 evaluates our algorithm through simulation. We summarize related work in Section 5 and then conclude this paper with future work in Section 6.

\section{Problem formulation}

The goal in this paper is to choose each sensor's sensing schedule in order to maximize the lifetime of a sensor network, while ensuring all intruding targets are detected before they reach protection points. For clarity, this section explains the basic idea of virtual scanning, using one road segment, and then we extend our design to arbitrary road networks in Section 3.

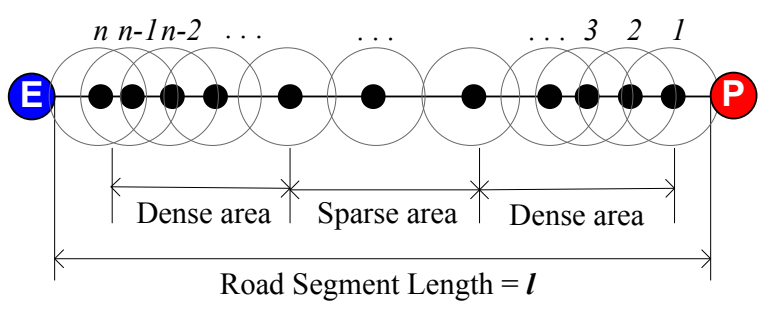

Fig. 2. Randomized Linear Deployment

\subsection{Virtual Scanning for Surveillance}

We assume $n$ sensors are randomly placed on a road segment of length $l$. Each sensor has a conservative sensing circle of radius $r$, which is long enough to cover the width of the road. This assumption holds true for most commercially available sensors (e.g., PIR sensors can detect moving car 60 100 feet away). Therefore, we can represent sensing coverage using a linear sensor network model as shown in Figure 2, where $n$ sensors are linearly placed. At the moment, let the left end of the road segment be the entrance point $E$ of targets and the right end of the road segment be the protection point $P$.

Let $w$ be the minimum working time needed by a sensor in order that the sensor can reliably detect a target over multiple samplings. Let $v$ be a maximum target speed. Suppose that targets enter only from the entrance point and move towards the protection point. In this scenario, we can use the traditional full coverage algorithms where sensors turn on all the time. We call this approach the Always-Awake.

A better design can be built based on the observation that it takes at least $l / v$ seconds for a target to pass a road segment of length $l$ at a maximum speed $v$. Therefore, all sensors in the road segment can sleep together for $l / v$ seconds, which is defined as silent time of the road network. After this silent time, all nodes wake up simultaneously for detection. We call this approach Duty Cycling.

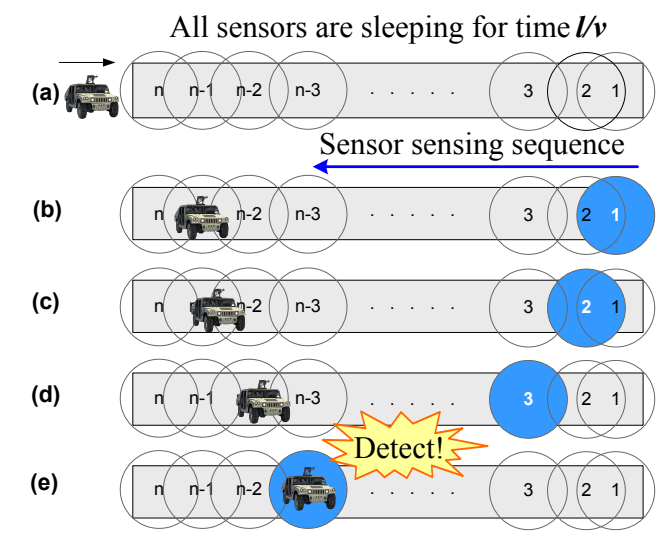

\section{Fig. 3. Sensor Sensing Sequence}

Based on the fact that targets move only along the roadways, we propose a new design called Virtual Scanning. As shown in Figure 3, after all sensors sleep for $l / v$ seconds, we turn on sensors one by one for working time $w$ from the rightmost sensor $s_{1}$ toward the leftmost one $s_{n}$. Clearly, this wave of sensing activities guarantees the detection and allows additional sleeping time for individual sensors. Compared with Duty Cycling, this additional sleeping time is obtained by the fact that all sensors but one can sleep during the scan. We note that the direction of a virtual scan shall be from the protection point to the entrance point. The virtual scan of the opposite direction (i.e., from the entrance point to the protection point) cannot guarantee target intrusion detection, if a very fast target enters right after the beginning of the networkwide silent time.

\subsection{Analytical Network Lifetime Comparison}

To understand key design parameters, this section compares analytically the network lifetime among the Always-Awake, Duty Cycling and Virtual Scanning methods. For clarity, we summarize the notation in Table 1 and overall analytical results in Table 2. Always-Awake \& Duty Cycling: For the Always-Awake approach, the network lifetime $T_{n e t}$ is the same as $T_{\text {life }}$, because sensors work continuously without sleeping. For the Duty Cycling approach, the network lifetime $T_{n e t}$ is the number of periods 
TABLE 2

Performance Analysis for Three Approaches

\begin{tabular}{|c|c|c|c|c|}
\hline Approach & Sleeping $\left(T_{\text {sleep }}\right)$ & Working $\left(T_{\text {work }}\right)$ & Network Lifetime $\left(T_{\text {net }}\right)$ & Avg. Detection Time \\
\hline Always-Awake & 0 & $T_{l i f e}$ & $T_{\text {life }}$ & 0 \\
\hline Duty Cycling & $\frac{l}{v}$ & $w$ & $\left\lfloor\frac{T_{l i f e}}{w}\right\rfloor\left(w+\frac{l}{v}\right)$ & $\frac{l^{2}}{2 v(w v+l)}$ \\
\hline Virtual Scanning & $(n-1) w+\frac{l}{v}$ & $w$ & $\left\lfloor\frac{T_{l i f e}}{w}\right\rfloor\left(n w+\frac{l}{v}\right)$ & $\frac{l}{2 v}$ \\
\hline
\end{tabular}

TABLE 1

Notation of Parameters for Analysis

\begin{tabular}{|l|l|}
\hline Parameter & Definition \\
\hline$T_{\text {life }}$ & $\begin{array}{l}\text { Lifetime that a sensor can work continuously } \\
\text { corresponding to its energy budget. }\end{array}$ \\
\hline$T_{\text {net }}$ & Sensor network lifetime. \\
\hline$T_{\text {work }}$ & $\begin{array}{l}\text { Working time that a sensor needs to work for } \\
\text { reliable detection. Normally } T_{\text {work }}=w .\end{array}$ \\
\hline$T_{\text {sleep }}$ & Sleeping time of each sensor. \\
\hline$T_{\text {scan }}$ & $\begin{array}{l}\text { Scan time that a virtual scan wave moves along } \\
\text { the road segment. } T_{\text {scan }}=n w .\end{array}$ \\
\hline$T_{\text {silent }}$ & $\begin{array}{l}\text { Silent time that the whole sensor network remains } \\
\text { silent; that is, time that a target passes through the } \\
\text { road segment of length } l . T_{\text {silent }}=l / v .\end{array}$ \\
\hline$T_{\text {period }}$ & $\begin{array}{l}\text { Schedule period of the sensor network. } \\
T_{\text {period }}=T_{\text {scan }}+T_{\text {silent }} .\end{array}$ \\
\hline
\end{tabular}

$\left\lfloor\frac{T_{l i f e}}{w}\right\rfloor$ multiplied by the length of the period $T_{\text {period }}$ (i.e., the sum of the silent time $\frac{l}{v}$ and the working time $w$ ):

$$
T_{n e t}=\left\lfloor\frac{T_{l i f e}}{w}\right\rfloor\left(\frac{l}{v}+w\right)
$$

Virtual Scanning: In the Virtual Scanning, the network lifetime $T_{n e t}$ is the number of periods $\left\lfloor\frac{T_{l i f e}}{w}\right\rfloor$ multiplied by the period length $T_{\text {period }}$. $T_{\text {period }}$ is the sum of the scan time $n w$ and silent time $\frac{l}{v}$ as shown in Figure 4. Therefore, we have:

$$
T_{\text {net }}=\left\lfloor\frac{T_{\text {life }}}{w}\right\rfloor\left(T_{\text {scan }}+T_{\text {silent }}\right)=\left\lfloor\frac{T_{\text {life }}}{w}\right\rfloor\left(n w+\frac{l}{v}\right)
$$

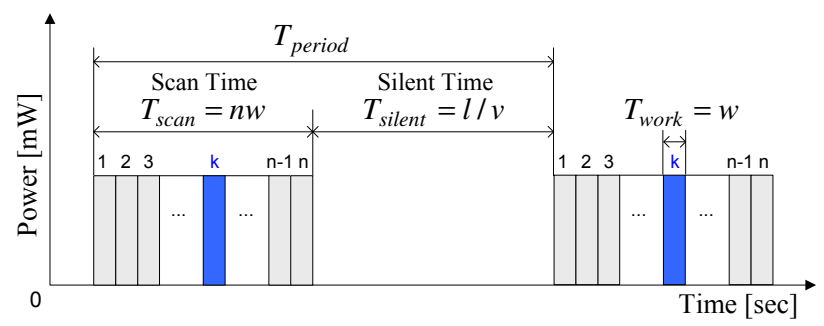

Fig. 4. Scheduling Time Diagram for Node $k$

Figure 5 shows the comparison of lifetime among these three approaches. For example, for $w=1 \mathrm{sec}$, Virtual Scanning has the lifetime of 30 hours, Duty Cycling 3.2 hours, and Always-Awake 0.14 hour; Virtual Scanning has 9.4 times lifetime of Duty Cycling and 214 times lifetime of Always-Awake.

\subsection{Analytical Detection Time Comparison}

This section compares the average detection time after a target entering a road segment among the Always-Awake, Duty Cycling and Virtual Scanning methods.

Always-Awake \& Duty Cycling: For Always-Awake, since a target is detected as soon as it enters the road segment, the average
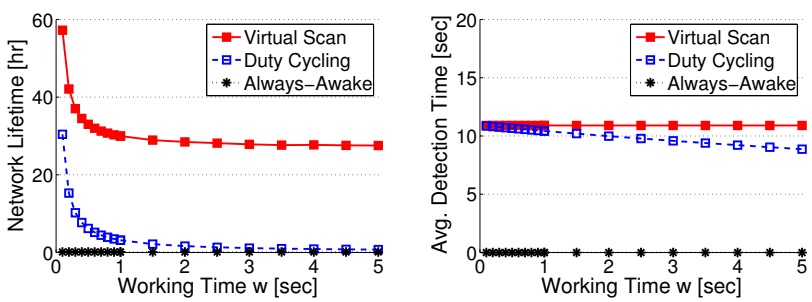

Fig. 5. Performance Comparison according to Working Time

detection time is zero. For the Duty Cycling, if a target enters during the working period, detection time is zero. On the other hand, if a target enters during the silent time, average detection time is half of the silent time $l /(2 v)$. The percentage of silent time within a period is $l /(w v+l)$, therefore, the overall average detection time of the Duty Cycling approach is $l^{2} /(2 v(w v+l))$. Virtual Scanning: We suppose that $n$ sensors are deployed on a road segment, so each sensor covers the length of $l / n$ in average. Also, we suppose that target speed is $v$ and a target can arrive at any time; that is, the arrival time is uniformly distributed. A target can arrive either during scan time or silent time. We analyze separately the average detection time for each period and then combine them to obtain overall expected delay $l /(2 v)$. Please refer to Appendix A for detailed derivation; note that the average detection time for bounded variable target speed is also derived in Appendix B.

Figure 5 shows the comparison of average detection time among the three approaches. Virtual Scanning detects with a constant delay $l /(2 v)$ regardless of working time $w$. On the other hand, the average detection time of the Duty Cycling tends to decrease slowly while working time $w$ increases. The Always-Awake method detects without any delay. For example, for working time $w=0.1 \mathrm{sec}$, Virtual Scanning has similar performance with Duty Cycling, about $10.9 \mathrm{sec}$. For working time $w=5 \mathrm{sec}$, the Virtual Scanning detects target within $10.9 \mathrm{sec}$ in average and the Duty Cycling does within $8.87 \mathrm{sec}$; the average detection delay ratio between the Virtual Scanning and the Duty Cycling is 1.23. However, the ratio of the Virtual Scanning's network lifetime to the Duty Cycling's network lifetime is 37, as shown in Figure 5. Thus, even though the average detection time increases slightly with Virtual Scanning, the benefit of network lifetime is quite remarkable.

\subsection{Configuring VISA for Better Delay and Longer Life- time}

As a reminder, when the network silent time $T_{\text {silent }}$ is equal to or smaller than $l / v$, target detection is guaranteed. Basic VISA design uses $l / v$ as the network silent time $T_{\text {silent }}$. However, if a smaller silent time $T_{\text {silent }}$ is used, it is possible to detect the target not only faster but also with less energy than the Duty Cycling algorithm. 

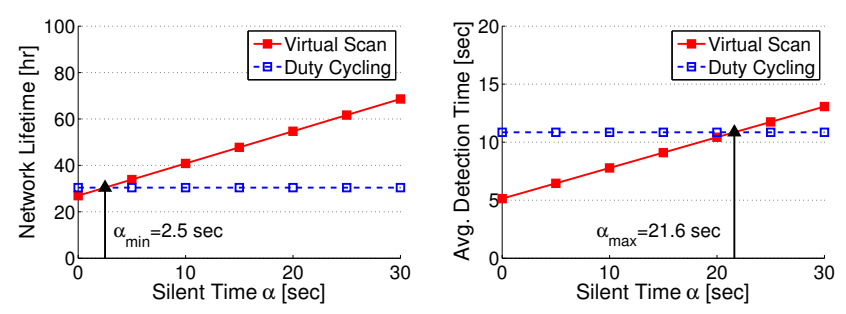

Fig. 6. Performance Comparison under Different $\alpha$ Values

Let $T_{\text {silent }}=\alpha$ for $\alpha \in[0, l / v]$. In order to outperform Duty Cycling in both network lifetime and average detection delay, we shall satisfy the following inequalities:

$$
\begin{array}{lll}
\text { Virtual Scanning } & & \text { Duty Cycling } \\
\left\lfloor\frac{T_{l i f e}}{w}\right\rfloor(n w+\alpha) & \geq\left\lfloor\frac{T_{l i f e}}{w}\right\rfloor\left(w+\frac{l}{v}\right) \\
\frac{l(n w+\alpha)}{2(n w v+l)} & \leq \frac{l^{2}}{2 v(w v+l)}
\end{array}
$$

Solving the above inequalities, we have:

$$
\max \left\{\frac{l}{v}-(n-1) w, 0\right\} \leq \alpha \leq \min \left\{\frac{l(n w v+l)}{v(w v+l)}-n w, \frac{l}{v}\right\}
$$

When $\alpha$ falls into this range, Virtual Scanning has better performance than Duty Cycling in both the average detection time and network lifetime. For example, as shown in Figure 6, for $w=0.1 \mathrm{sec}$, when $\alpha$ is less than $\alpha_{\max }=21.6 \mathrm{sec}$, the average detection time of Virtual Scanning is shorter than that of Duty Cycling. Also, when $\alpha$ is greater than $\alpha_{\min }=2.5 \mathrm{sec}$, Virtual Scanning's lifetime is longer than Duty Cycling's. Thus, the range of $\alpha$ achieving better detection delay and lifetime is [2.5, 21.6] sec. We note the results here only illustrate the idea. Detailed study on the performance effect of $\alpha$ is presented in evaluation Section 4.3.

\section{Virtual Scanning Algorithm Design}

For the sake of clarity, the previous section presents the basic idea using one road segment. In the rest of the paper, we demonstrate how to apply the virtual scanning to road networks with arbitrary topology. This section is organized as follows: Section 3.1 lists definitions and assumptions used in VISA. Section 3.2 describes the scheduling algorithm, and Section 3.3 presents the hole handling algorithm.

\subsection{Definitions and Assumptions}

Definition 3.1 (Road Network Graph): Let Road Network Graph be $G=(V, E)$, where $V=\left\{v_{1}, v_{2}, \ldots, v_{n}\right\}$ is a set of intersections, entrance points, and protection points in the road network under surveillance, and $E=\left[e_{i j}\right]$ is a matrix of road segment length $e_{i j}$ for vertices $v_{i}$ and $v_{j}$. Figure 7 shows a graph $G$ corresponding to the road network in Figure 1. Note that the entrance point set and the protection point set are not static. These two sets can be changed either on demand for military maneuvers or to deal with sensing holes in Section 3.3.

Definition 3.2 (Network Lifetime): Let Network Lifetime be the duration from the starting of a sensor network for surveillance until a target can possibly reach one of the protection points without detection. In other words, lifetime ends when there exists a possible breach path between an entrance point to a protection

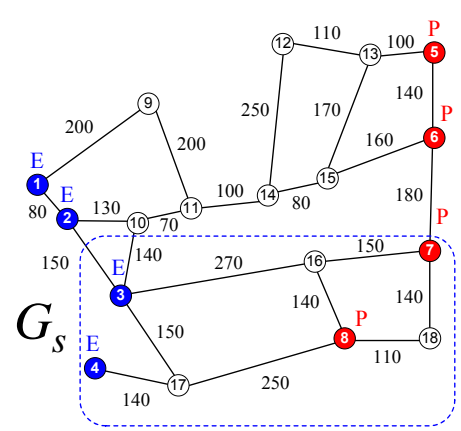

Fig. 7. Road Network Graph $G$

point. Note that for the target, we cannot differentiate between energy vehicles and our vehicles from detection since binary sensors are used.

Definition 3.3 (VISA Scheduler): Let VISA Scheduler be a sink node that initiates the sensing scheduling algorithm.

The VISA design is based on the following assumptions:

- Road map and locations of sensor nodes are known to VISA Scheduler. The sensor location can be obtained through localization schemes [5].

- Sensors are roughly time-synchronized at tens of millisecond level. It can be easily achieved because existing solutions [6], [7] can achieve microsecond level accuracy.

- Sensors only have simple sensing devices for binary target detection, such as PIR sensors [8]. No sophisticated hardware is available.

- The circular sensing model is used in the conservative way using the minimum sensing range. For irregular sensing modeling, SAM [9] can be used to explore in-situ sensing irregularity.

- One of existing low-duty-cycle data forwarding schemes, such as DSF [10] and DESS [11] is used to deliver nodes' locations and target detection results to the VISA scheduler.

- Targets move only along predefined roads with the bounded maximum speed.

\subsection{VISA Scheduling on Road Network}

This section presents the design of virtual scanning, including schedule establishment and dissemination.

\subsubsection{Establishment of Working Schedule}

For clarity in presentation, we use the subgraph $G_{s}$ of the graph $G$ shown in Figure 7 where the edge weight means the physical distance of the road segment. First, we will consider a road network with one entrance and one protection point at first, and then will consider a road network with multiple entrances and multiple protection points. Also, for now, we assume that no sensing holes exist in the middle of roadways where targets cannot be detected due to the non-existence of sensors. The sensing hole handling will be discussed in Section 3.3.

Figure 8 shows the snapshots of virtual scanning in the road network $G_{s}$ with one entrance $v_{4}$ labeled as $E$ and one protection point $v_{7}$ labeled as $P$; note that one edge $e_{3,4}$ of length 50 is added to $G_{s}$ in order to explain the virtual scanning. The virtual scan's propagation time on each road segment is the multiplication of the number of sensors and the individual working time $w$, 


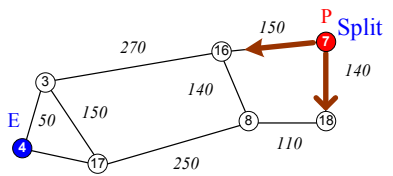

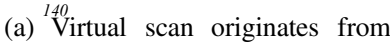
protection point $v_{7}$ and splits into two scans

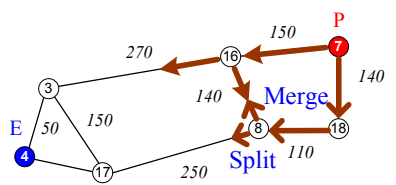

(c) ${ }^{140}$ Two scans from $v_{8}$ and $v_{16}$ merge at the road segment $\left(v_{8}, v_{16}\right)$ and stop

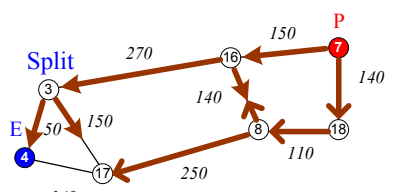

(e) Virtual scan arriving at $v_{3}$ splits into two scans towards $v_{4}$ and $v_{17}$

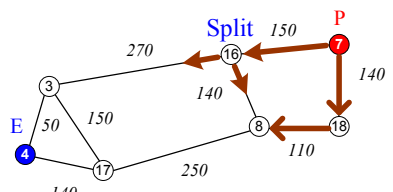

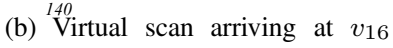
splits into two scans towards $v_{3}$ and $v_{8}$

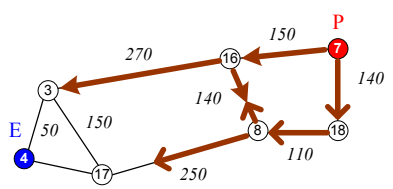

(d) 140 Two scans keep going towards intersections $v_{3}$ and $v_{17}$, respectively

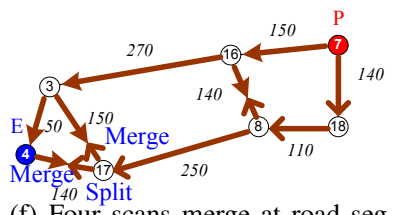

(f) Four scans merge at road segments $\left(v_{3}, v_{17}\right)$ and $\left(v_{4}, v_{17}\right)$, respectively

\subsubsection{Establishment of Sleeping Schedule}

The previous section discussed how to decide working schedule during the scan. This section explains how to compute the optimal sleeping length, i.e., the maximum duration sensors can sleep safely after working for $w$ seconds while guaranteeing the detection.

Figure 9(a) shows the virtual scanning in an arbitrary road network. Let $P=\left\{p_{1}, \ldots, p_{n}\right\}$ be the set of protection points. Let $E=\left\{e_{1}, \ldots, e_{m}\right\}$ be the set of entrance points. As discussed before, a period $T_{\text {period }}$ consists of (i) silent time $T_{\text {silent }}$ during which the whole network is turned off and (ii) scan time $T_{\text {scan }}$ during which scan waves propagate across the network. Since a sensor only works for fixed $T_{\text {work }}=w$ seconds every $T_{\text {period }}$, the longer $T_{\text {period }}$ is, the better energy efficiency we have. Therefore, we shall identify the maximum $T_{\text {period }}$ value that can guarantee the detection. Before this optimization, we define two important concepts as below:

Definition 3.4 (The Shortest Scanning Path): The Shortest Scanning Path $p_{\text {scan }}(i, j)$ is the shortest-delay path for wave propagation from $v_{i}$ to $v_{j}$ on the graph $G$, where $v_{i} \in P$ and $v_{j} \in E$. Let $l_{\text {scan }}(i, j)$ be the number of sensors along the path $p_{\text {scan }}(i, j)$. Therefore, the Shortest Scanning Time $T_{\text {scan }}(i, j)$

Fig. 8. Virtual Scanning on Road Network for Working Schedule Establishment

instead of the physical distance of a road segment. As shown in Figure 8 , by turning on sensors along roads consecutively, virtual scanning waves propagate along multiple routes simultaneously, split at intersections, and disappear when two waves encounter each other in a road segment. Note that once a virtual scanning wave arrives at entrance $E$, it keeps propagating to the rest of the road network. This scanning gurantees the detection of all of the mobile targets in the road network.

In the case of multiple entrance and protection points, scan operation is similar, except that multiple protection points initiate scanning at the same time. Because the waves merge in the middle of road segments during virtual scanning (as shown in Figure 8(c)), regardless the number of protection points and the locations of the sensors, each sensor only works for $w$ seconds per scan, which is a nice feature for energy balance. Clearly, the scan wave arrival time for each sensor can be easily computed with All-Pairs Shortest Path algorithm, such as Floyd-Warshall algorithm [12]. We note the scan wave arrival time decides the working schedule of a sensor node. In other words, a sensor shall start to work for $w$ seconds after a virtual scanning wave arrives.

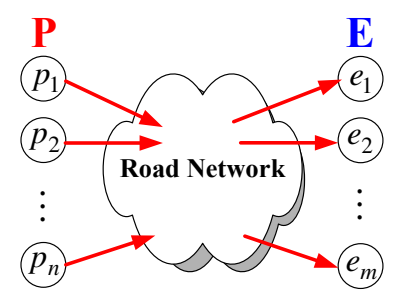

(a) Virtual Scanning on an Arbitrary Road Network with Protection Points and Entrance Points

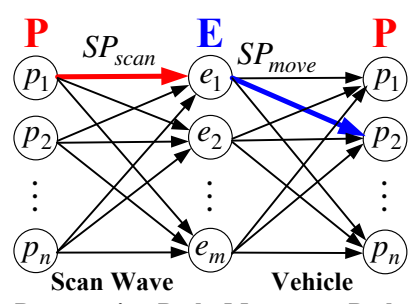

Propagation Path Movement Path (b) Sleeping Time Computation considering the Shortest Scanning and Movement Paths

Definition 3.5 (The Shortest Movement Path): The Shortest Movement Path $p_{\text {move }}(i, j)$ is the shortest-distance path between vertices $v_{i}$ and $v_{j}$ on the graph $G$ where $v_{i} \in E$ and $v_{j} \in P$. Let $l_{\text {move }}(i, j)$ be the shortest distance of $p_{\text {move }}(i, j)$. Therefore, the Shortest Movement Time $T_{\text {silent }}(i, j)$ can be computed as $l_{\text {move }}(i, j) / v_{\max }$, where $v_{\max }$ is maximum target speed. We note that all of the sensors along the path $p_{\text {move }}(i, j)$ can sleep together for the silent time $T_{\text {silent }}(i, j)$.

These two shortest paths $p_{\text {scan }}(i, j)$ and $p_{\text {move }}(i, j)$ for all pairs of vertices can be computed based on $G$ by the All-Pairs Shortest Paths algorithm, such as Floyd-Warshall algorithm.

An important principle of computing the sleeping time is that all of vehicles entering during the sleeping time must be detected before their arrival to the protection points. Once a virtual scan wave originating from the protection points has swept an entrance point, the paths from this swept entrance point to the protection points are vulnerable to the target intrusion. This is because the swept paths are not swept again until the next scan period.

It is noted that we can guarantee detection by setting $T_{\text {period }}$ as the sum of all-pair minimum scanning time and all-pair minimum movement time. However, the resulting $T_{\text {period }}$ is shorter than the optimal value, (i) because an intruding target could have to travel a longer route from an entrance point with the earliest scan arriving time than the shortest route with all-pair minimum movement time, or (ii) because it could have to wait until a late scan arrives before it can travel along the shortest route with all-pair minimum movement time, especially when sensors are non-uniformly placed across a network.

We claim that a longer safe period $T_{\text {period }}$ can be obtained as the minimum sum of the scanning time from $v_{i}$ to $v_{j}$ and the vehicle movement time from $v_{j}$ to $v_{k}$, for $v_{i}, v_{k} \in P$ and $v_{j} \in E$ than the sum of all-pair minimum scanning time and all-pair minimum movement time. In order to prove this claim,

Fig. 9. Virtual Scanning on Road Networks 
a three-column graph is introduced as shown in Figure 9(b). The edges between the first and second columns denote the time for wave propagation and the edges between the second and third columns denote the time for target movement. To compute a safe period $T_{\text {period }}$, we need to identify the shortest path from any vertex in the first column to any vertex in the third column. Without loss of generality, suppose $p_{1} \Rightarrow e_{1} \Rightarrow p_{2}$ is the shortest path where $p_{1} \Rightarrow e_{1}$ is the shortest scanning path from $p_{1}$ to $e_{1}, e_{1} \Rightarrow p_{2}$ is the shortest movement path from $e_{1}$ to $p_{2}$, and the sum of the scanning time and the movement time for these two paths is the minimum among all of the possible ones. Once the virtual scan arrives at the entrance point $e_{1}$ with a delay of $T_{\text {scan }}\left(p_{1}, e_{1}\right)$, the path from the entrance point $e_{1}$ to the protection point $p_{2}$ becomes vulnerable, if the network remains silent for more than $T_{\text {silent }}\left(e_{1}, p_{2}\right)$. Thus, to prevent a target from reaching the protection point $p_{2}$ without detection, another scan wave must be generated from the protection point $p_{2}$ after $T_{\text {silent }}\left(e_{1}, p_{2}\right)$. Thus, the safe period is $T_{\text {period }}=T_{\text {scan }}\left(p_{1}, e_{1}\right)+T_{\text {silent }}\left(e_{1}, p_{2}\right)$. Note that $p_{1} \Rightarrow e_{1}$ and $e_{1} \Rightarrow p_{2}$ are not necessarily all-pair minimum scanning path and all-pair minimum movement path simultaneously. Therefore, our claim is proved. Consequently, the sleeping time is $T_{\text {sleep }}=T_{\text {period }}-T_{\text {work }}$, because each sensor must work for its duty cycle $T_{\text {work }}=w$ seconds per period.

Now, we can formally define the optimization problem of the sleeping time. Suppose that sensors are randomly deployed into the target road network with a uniform sensor density; note that since the number of sensors is proportional to the road segment length, the shortest scanning path is the same as the reverse of the shortest movement path. Let $T_{\text {sleep }}(i, j, k)=T_{\text {scan }}(i, j)+$ $T_{\text {silent }}(j, k)-T_{\text {work }}$ for $v_{i}, v_{k} \in P$ and $v_{j} \in E$ where $T_{w o r k}=w$. The optimal sleeping time is chosen as follows:

$$
T_{\text {sleep }} \leftarrow \min _{\substack{v_{i}, v_{k} \in P \\ v_{j} \in E}} T_{\text {sleep }}(i, j, k)
$$

Obviously, the searching for an optimal sleeping time is done in polynomial time $O\left(m n^{2}\right)$. It is noted that only under a uniform sensor density, the formulation in Eq. 4 guarantees the optimal sleeping time. Even for a non-uniform sensor density, this formulation provides a good sleeping time close to the optimal one. We leave the optimization for this non-uniform sensor density as future work.

Once the sleeping time value is computed by VISA scheduler, it piggybacks in the counter message discussed in Section 3.2.3 and is disseminated to all the sensors in the network. If the VISA scheduler changes the locations of protection and entrance points dynamically, it only needs to re-calculate a new sleeping time and re-disseminate it.

\subsubsection{Decentralized Implementation}

In a centralized implementation, a VISA scheduler calculates the working schedules for all sensors and disseminate the results, which leads to far more messages than necessary ones. Actually the scan wave arrival time for each sensor can be calculated in a decentralized way. During the initialization phase, all sensors are awake. The sensors at the protection points generate short messages containing a counter with value initialized to one, and pass them to their immediate neighboring sensors. The neighboring sensors only record the minimum counter value ever seen (i.e.,

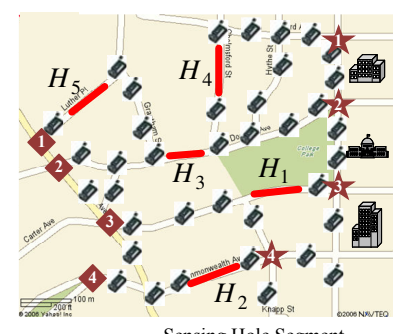

(a) Sensing Hole Segment Road Network: $H_{i}=\left(h_{j}, h_{k}\right)$

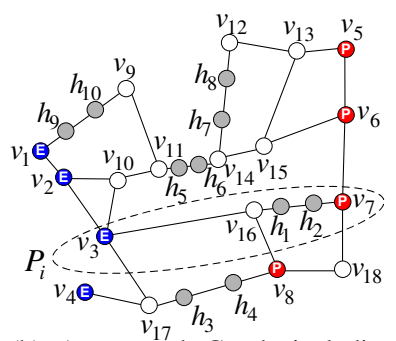

(b) Augmented Graph including Sensing Holes: $G_{a}=\left(V_{a}, E_{a}\right)$

\section{Fig. 10. Augmentation of Road Network Graph for Holes}

discard the rest of messages arriving late), increment the counter, and then relay the message to their neighboring sensors. If a sensor is located at a road intersection, it duplicates and relays multiple copies of messages to all its neighboring nodes except the one it received the message from. In this way, the sensors can decide their sensing scanning order (i.e., the minimum counter value) in the distributed way. Given a sensing order of $K$, a node shall start to work at time $K w$ and stop at time $(K+1) w$.

For the sleeping schedule, given the sensor density and the road network along with the entrance and protection points, a conservative sleeping time can initially be estimated by VISA Scheduler; note that sensor density $\rho$ is defined as the number of sensors within the sensing range $2 r$ where $r$ is the sensing radius. For example, under a uniform sensor density $\rho$, we can estimate a conservative sleeping time as scanning time + movement time working time $=\alpha\left(\frac{l}{2 r} \rho w+\frac{l}{v}-w\right)$ where the shortest path's length is $l$, the sensing radius is $r$, the working time is $w$, the maximum vehicle speed is $v$, and the coefficient is $\alpha$ (e.g., 0.9). This sleeping time piggybacks in the counter message for the setup of sensors' working schedules in a decentralized way above. After this setup of working schedules, the number of sensors per road segment is reported to VISA Scheduler to determine an optimal sleeping time. This optimal sleeping time will replace the previous conservative sleeping time later.

Up to now, the sensors know when to wake up in order to create virtual scanning (i.e., Working Schedule in Section 3.2.1) and how long they can safely sleep with optimal efficiency (i.e., Sleeping Schedule in Section 3.2.2). Note that if the entrance point set $E$ and the protection point set $P$ are changed either on demand for military maneuvers or to deal with sensing holes in Section 3.3, both Working Schedule and Sleeping Schedule are performed for these updated sets.

\subsection{Handling of Sensing Holes}

We have so far discussed the sensor working schedule and sleeping schedule, assuming balanced energy and no initial sensing holes. In this section, we discuss the handling of sensing holes that can exist after the sensor deployment and that can occur due to sensor failure or energy depletion. Note that for such sensing holes, we can use sensing hole detection schemes previously studied [13], [14]. As shown in Figure 10(a), there exist five sensing hole segments (i.e., $H_{1}, \ldots, H_{5}$ ) that cannot be covered by sensors in the given road network graph. Our idea to deal with these initial hole segments is that we make an augmented graph by adding the endpoints of the hole segments (called hole endpoints) 
as shown in Figure 10(b). Note that these initial hole segments and the corresponding hole endpoints can be identified by VISA Scheduler since sensors report their locations to VISA Scheduler at the initialization phase. To ensure the protection, we treat the hole endpoints as either pseudo entrance points or pseudo protection points. The hole handling problem is, therefore, reduced to a labeling problem of hole endpoints.

Problem Definition: How to optimally determine the role of each hole endpoint (i.e., label as either entrance point or protection point) in order to achieve the maximum sleeping time, leading to the maximization of the sensor network lifetime.

In the rest of this section, we present an optimal labeling algorithm for hole handling.

\subsubsection{Initial Sensing Holes}

In reality, there is high probability that some road segments are not covered by sensors even though many sensors are randomly deployed on road network as shown in Figure 10(a). We define these uncovered road segments as the initial sensing hole segments; note that each sensing hole segment consists of two hole endpoints.

Suppose that $n$ hole endpoints occur under a uniform sensor density. With an exhaustive search, $2^{n}$ cases are required to investigate. This means the time complexity of $O\left(2^{n}\right)$. Since this complexity is intractable, we need an improved way to achieve an optimal labeling for hole endpoints.

We explain here the idea with a simplified example; Figure 10(b) shows one roadway $P_{i}$ consisting of $v_{3}, v_{16}$, and $v_{7}$ and a hole segment $H_{1}$ with hole endpoints $h_{1}$ and $h_{2}$, which are closer to a protection point $v_{7}$ than an entrance point $v_{3}$. If two hole endpoints $h_{1}$ and $h_{2}$ are labeled differently, this short hole segment determines the shortest sleeping time. To avoid this, $h_{1}$ and $h_{2}$ should have the same type of label. Furthermore, since $h_{1}$ and $h_{2}$ near the protection point $v_{7}$, in order to get a longer sleeping time, they should be labeled as protection points.

Conceptually, when labeling hole endpoints, we should label each hole endpoint with the same label as the closest point already labeled. Rationale behind this insight is: the maximization of the path distance between the entrance points and protection points leads to a maximum sleeping time according to Eq. 4.

Formally, let $H$ be the set of hole endpoints such that $H=$ $\left\{h_{1}, h_{2}, \ldots, h_{k}\right\}$. Let $E$ be the set of entrance points and $P$ be the set of protection points. Let $L_{E}$ be entrance label and $L_{P}$ be protection label. We can label the holes in $H$, by partitioning $H$ into two disjoint subsets (called clusters) Entrance Cluster $\left(C_{E}\right)$ and Protection Cluster $\left(C_{P}\right)$. Asano et al. proposed such a clustering algorithm for a farthest k-partition based on Minimum Spanning Tree (MST) [15], giving an optimal clustering to maximize the inter-cluster distance. We extend Asano's Clustering for sensing hole labeling.

Figure 11 illustrates the main idea. Let $\operatorname{dist}\left(C_{E}, C_{P}\right)$ be the inter-cluster distance between $C_{E}$ and $C_{P}$. Our objective is to partition the set $H$ into two disjoint sets $C_{E}$ and $C_{P}$ such that the inter-cluster distance between $C_{E}$ and $C_{P}$ is maximized. The initial inter-cluster distance is $\operatorname{dist}\left(C_{E}, C_{P}\right)=d_{0}$, as shown in Figure 11(a). In this example, suppose that two hole clusters $h_{1}$ and $h_{2}$ are the closest pair of two clusters. In this case, these

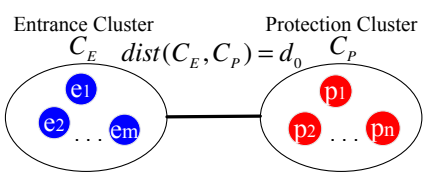

h1 $h_{2} h_{3} h_{4} \quad \ldots h k$ k Hole Clusters (a) Initial Clusters: Entrance Cluster (b) The closest pair of $h_{1}$ and $h_{2}$ $C_{E}$, Protection Cluster $C_{P}$, and $k$ are merged into cluster $H_{1}$ labeled as Hole Clusters $h_{s}$ for $s=1 . . k \quad$ unknown

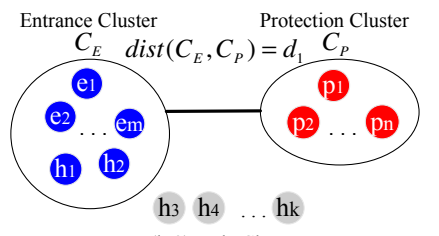

(k-2) Hole Cluster

(c) The closest pair of $C_{E}$ and $H_{1}$ (d) After clustering, all hole clusters are merged into $C_{E}$ with $H_{1}$ labeled are merged into either $C_{E}$ or $C_{P}$, that as entrance label $L_{E}$
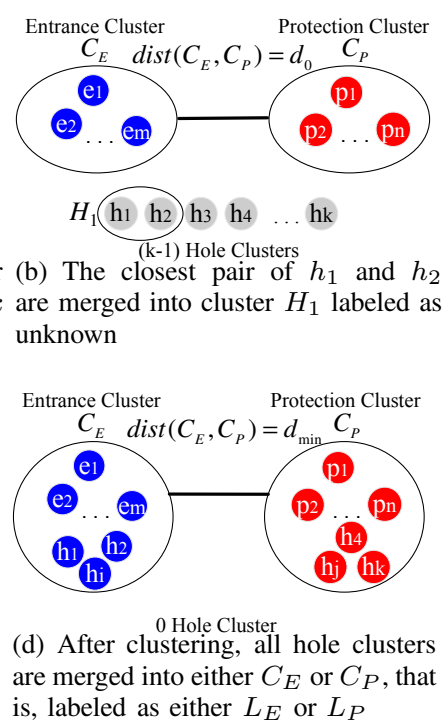

\section{Fig. 11. Clustering for Sensing Hole Labeling}

hole clusters are merged into one hole cluster $H_{1}$ with the same, unknown label, as shown in Figure 11(b). The reason two clusters $h_{1}$ and $h_{2}$ are merged into one hole cluster with the same label is to let the inter-cluster distance between $C_{E}$ and $C_{P}$ be maximized. Otherwise, the inter-cluster distance between $h_{1}$ and $h_{2}$ can be the inter-cluster distance shorter than the initial inter-cluster distance $\operatorname{dist}\left(C_{E}, C_{P}\right)=d_{0}$. As shown in Figure 11(c), two clusters $C_{E}$ and $H_{1}$ are the closest pair, so $H_{1}$ is merged into $C_{E}$ with hole endpoints $h_{1}$ and $h_{2}$ labeled as entrance. In this way, we can cluster all of the hole endpoints into either $C_{E}$ or $C_{P}$ to maximize the inter-cluster distance $\operatorname{dist}\left(C_{E}, C_{P}\right)$, as shown in Figure 11(d). Similar to Asano's algorithm [15], our clustering gives an optimal hole labeling because it satisfies the greedy choice property and optimal substructure [12].

As an important difference from Asano's Clustering, during the clustering, we maintain multiple hole clusters $H_{i}$ labeled as unknown in addition to one Entrance Cluster $C_{E}$ and one Protection Cluster $C_{P}$. Through the MST construction, we merge one hole cluster $H_{i}$ to either $C_{E}$ or $C_{P}$ such that the inter-cluster distance between $C_{E}$ and $C_{P}$ is maximized. We call this new labeling algorithm the MST-based Labeling.

\subsubsection{Sensing Holes due to Energy Depletion or Failure}

In the previous section, we discussed the initial sensing hole issue. However, since in reality, the sensors deployed on road network may not have the same amount of energy initially, we need to consider the sensing holes caused by this unbalanced sensor energy budget. Also sensor could fail over time. We can deal with these sensing holes in the same way as with the initial holes; we can either completely relabel all holes or incrementally label new holes by using MST-based Labeling. The former is optimal, but the latter introduces less computation.

To detect the failure of sensors due to energy depletion, the sensors can regularly report their existence to VISA Scheduler. Also, in a distributed way, the neighboring nodes can exchange their health status with each other in a regular basis. We leave this kind of fault node detection as future work, because in VISA, the fault node detection is not a key design component. 


\subsection{Handling of Detection Failure Probability}

In reality, there exist sensing errors in sensors. We need to relax the assumption that every vehicle within the sensing range of some sensors can be detected with probability one. Let $p$ be the probability of a success (called detection success probability) on each sensing for working time $w$. When the required networkwide detection probability for mobile targets is given as $P_{r e q}$ (e.g., 0.99 ) and the sensor's detection success probability is known as $p=0.9$, the question is how to schedule sensors in order to achieve the user-required detection probability $P_{r e q}$. The idea is to perform multiple sensing activities. For example, let $q$ be the probability of a failure (called detection failure probability) where $q=1-p$. When $p=0.9, q=0.1$, which means that there exists one detection missing among ten detection trials. Let $P_{r e q}=0.99$, which means that there exists one detection missing among one hundred detection trials. Assume that a mobile target is staying at a sensor's sensing range. In order to achieve $P_{r e q}=0.99$, the sensor needs to perform two consecutive sensing activities because the corresponding network-wide detection failure probability is $1-P_{\text {req }}=0.01$ and the detection failure of two consecutive sensing activities is $(1-q)^{2}=0.01$. Another way is for two sensors to perform their sensing activity simultaneously for the mobile target. The first approach is defined as temporal redundant sensing and the second as spatial redundant sensing. Our scheme to deal with the detection failure probability is the combination of these two approaches, defined as redundant sensing.

First, we explain the number of redundant sensing activities $N$ given $q$ and $P_{r e q}$. Let $\bar{P}_{r e q}$ be the network-wide detection failure probability such that $\bar{P}_{r e q}=1-P_{r e q}$. Suppose the sensing activities are independent and identically distributed (i.i.d.). The number $N$ can be computed as follows:

$$
q^{N} \leq \bar{P}_{r e q} \Rightarrow N=\left\lceil\log _{q} \bar{P}_{r e q}\right\rceil
$$

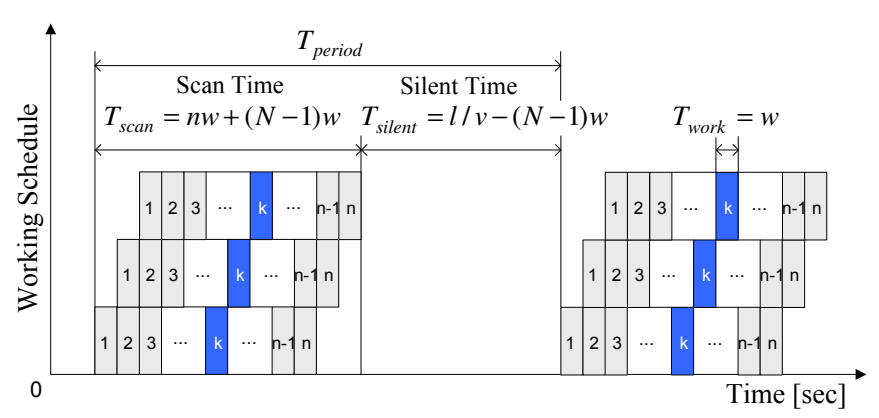

Fig. 12. Scheduling Time Diagram for Redundant Sensing

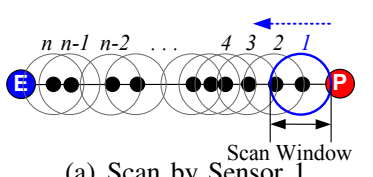

(a) Scan by Sensor 1

(c) Scan by Sensors 1,2 and 3

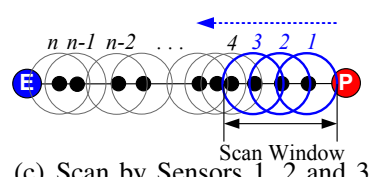

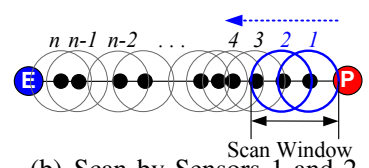

(b) Scan by Sensors 1 and 2

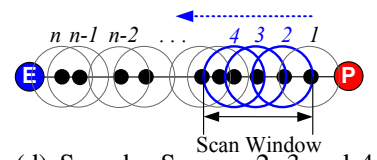

(d) Scan by Sensors 2,3 and 4
Fig. 13. Virtual Scanning for Redundant Sensing
Figure 12 shows the scheduling time diagram for the redundant sensing in the Virtual Scanning. As shown in the figure, each sensor performs $N$ sensing activities consecutively. For the redundant sensing, we perform $N$ virtual scans consecutively from the protection point towards the entrance point. To guarantee that a mobile target is scanned at least $N$ times, the silent time $T_{\text {silent }}$ is reduced to $\frac{l}{v}-(N-1) w$. This is because $N$ scans should be started before the mobile target reaches the protection point.

Figure 13 shows the virtual scanning for the redundant sensing where $N=3$, which is equivalent to the scheduling time diagram in Figure 12. The scan window is defined as the cluster of adjacent working sensors. Initially, as shown in Figure 13(a), when sensor $s_{1}$ is working, the size of the scan window is 1 . After the working time $w$, as shown in Figure 13(b), the size becomes 2 since two sensors $s_{1}$ and $s_{2}$ are working together. After another $w$, as shown in Figure 13(c), the size becomes 3 since three sensors $s_{1}, s_{2}$ and $s_{3}$ are working together. Finally, after another $w$, as shown in Figure 13(d), the scan window of size 3 is shifted to the left because the number $N$ of simultaneous working sensors is 3 .

For the Duty Cycling, each sensor performs $N$ sensing activities per its duty cycle in order to provide the same detection probability as with the Virtual Scanning. On the other hand, for the AlwaysAwake, no change is required for the redudant sensing, because it can already provide such a redudant sensing. We summarize the performance analysis for these three approaches in Table 3 in terms of the maximum network lifetime $T_{n e t}$. Note that the network lifetime is usually less than $T_{n e t}$ since a vehicle can pass the sensor network at any time without detection where each sensor can detect a vehicle with detection success probability $p$ per sensing trial; that is, because the sensor network tries to detect the vehicle $N$ times with the detection success probability $p$ per sensing trial, it can fail detecting the vehicle with probability $(1-p)^{N}$ for $N$ trials per duty cycle.

\subsection{Handling of Time Synchronization Error}

Up to this point, it is assumed that sensors in VISA system are roughly time-synchronized as long as there is no time gap between two neighboring sensors during the scan time for vehicle detection. Considering that many state-of-the-art solutions [6], [7] can provide sensors with the time synchronization at the microsecond level, we explain how to perform the virtual scanning to satisfy this assumption in this subsection.

Figure 14 illustrates the handling of the time synchronization by the overlap of the working schedules through the margin of sensing time. As shown in Figure 14(a), there exist time gaps among the working schedules of sensors $s_{k-1}, s_{k}$ and $s_{k+1}$. However, as shown in Figure 14(b), through the margin of the working time based on the maximum time synchronization error $\epsilon_{\max }$, the working schedules have time overlaps, guaranteeing no time gaps among the working schedules.

This detection guarantee can be explained in a more formal way as follows: Suppose that a maximum time synchronization error is known as $\epsilon_{\max }$, sensor $s_{k}$ is required to have a margin of $\epsilon_{\max }$ for its working start time $t_{k}^{s}$ and working end time $t_{k}^{e}$ such that the working schedule is $\left[t_{k}^{s}-\epsilon_{\max }, t_{k}^{e}+\epsilon_{\max }\right]$. This allows two adjacent sensors' working schedules to overlap even under maximum time synchronization errors. It can be explained that this working 
TABLE 3

Performance Analysis for Three Approaches in Redudant Sensing

\begin{tabular}{|c|c|c|c|}
\hline Approach & Sleeping $\left(T_{\text {sleep }}\right)$ & Working $\left(T_{\text {work }}\right)$ & Maximum Network Lifetime $\left(T_{n e t}\right)$ \\
\hline Always-Awake & 0 & $T_{l i f e}$ & $T_{l i f e}$ \\
\hline Duty Cycling & $\frac{l}{v}-(N-1) w$ & $N w$ & $\left\lfloor\frac{T_{l i f e}}{N w}\right\rfloor\left(N w+\frac{l}{v}-(N-1) w\right)$ \\
\hline Virtual Scanning & $(n-1) w+\frac{l}{v}-(N-1) w$ & $N w$ & $\left\lfloor\frac{T_{l i f e}}{N w}\right\rfloor\left(n w+\frac{l}{v}\right)$ \\
\hline
\end{tabular}
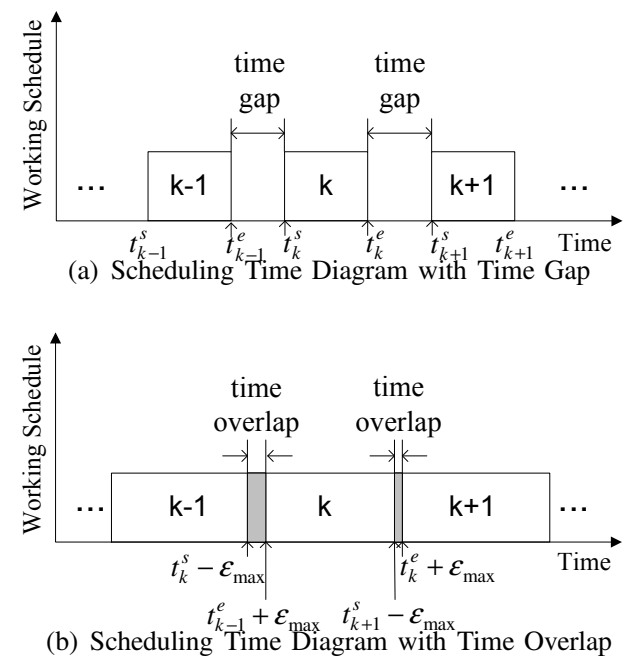

Fig. 14. Handling of Time Synchronization Error

schedule with a margin of $\epsilon_{\max }$ guarantees the target detection as follows: Suppose that the working time is $w$, the accurate working schedules of two adjacent sensors $s_{k}$ and $s_{k+1}$ are $\left[t^{*}, t^{*}+w\right]$ and $\left[t^{*}+w, t^{*}+2 w\right]$, respectively, and the their synchronization errors are $\epsilon_{k}$ and $\epsilon_{k+1} \in\left[-\epsilon_{\max }, \epsilon_{\max }\right]$, respectively. Let us consider the worst scenario for the maximum time gap between these two working schedules, that is, $\epsilon_{k}=-\epsilon_{\max }$ and $\epsilon_{k+1}=\epsilon_{\max }$. This setting makes the following working schedules: (i) $s_{k}$ 's working schedule $=\left[t^{*}-\epsilon_{\max }, t^{*}+w-\epsilon_{\max }\right]$ and (ii) $s_{k+1}$ 's working schedule $=\left[t^{*}+w+\epsilon_{\max }, t^{*}+2 w+\epsilon_{\max }\right]$. Now, we augment these two schedules with the maximum time synchronization error $\epsilon_{\max }$ for the safe detection as follows: (i) $s_{k}$ 's new working schedule $=\left[t^{*}-2 \epsilon_{\max }, t^{*}+w\right]$ and (ii) $s_{k+1}$ 's new working schedule $=$ $\left[t^{*}+w, t^{*}+2 w+2 \epsilon_{\max }\right]$. Thus, these new working schedules have no time gap, so the targets can be detected without missing. Note the sleeping time $T_{\text {sleep }}$ for sensor $s_{k}$ decreases by $2 \epsilon_{\max }$ since the working time $T_{\text {work }}$ becomes $w+2 \epsilon_{\max }$ in the schedule period $T_{\text {period. }}$. Duty Cycling also needs to adjust each sensor's working time and sleeping time by $w+2 \epsilon_{\max }$ in the same way as with Virtual Scanning. On the other hand, Always-Awake does not need this adjustment since it does not have any sleeping time.

\section{Performance Evaluation}

In this section, we analyze performance of VISA, comparing with other schemes for road network surveillance.

- Performance Metrics: We use network lifetime and average detection time as the performance metrics.

- Baselines: Since the road network surveillance is a new research area, to the best of our knowledge, there exist no other state-of-the-art sensing schemes for road network surveillance. We compare VISA with two approaches: Duty Cycling and Always-Awake.
- Parameters: In the performance comparison, we investigate the effect of the following three parameters: (i) working time, (ii) sensor density, (iii) time synchronization error, and (iv) detection failure probability. In addition, we reveal (i) the effect of sleeping time duration and (ii) the effect of sensing hole labeling.

Simulation uses the map of a real road network as shown in Figure 7. For vehicle mobility, vehicles arrive at the specified entrances of the road network and randomly choose one protection point as destination, moving towards the destination via the shortest path. The vehicle arrival time is uniformly distributed during the system lifetime with mean inter-arrival time $60 \mathrm{sec}$. The system parameters are selected based on a typical military scenario [16]. Unless mentioned otherwise, the default values in Table 4 are used. Based on these settings, we implemented our own event-driven simulator for evaluation.

\section{TABLE 4 \\ Simulation Configuration}

\begin{tabular}{|c|c|}
\hline Parameter & Description \\
\hline $\begin{array}{l}\text { Sensing range } \\
R\end{array}$ & $\begin{array}{l}R=2 r=20 \text { meters (i.e., } 66 \text { feet) where } r \text { is sensing } \\
\text { radius. }\end{array}$ \\
\hline $\begin{array}{l}\text { Energy budget } \\
b\end{array}$ & $\begin{array}{l}b \sim N\left(\mu_{b}, \sigma_{b}\right) \text { where } \mu_{b}=50 \text { kilo-joule }(\mathrm{kJ}) \text { and } \\
\sigma_{b}=\{0,2, \ldots, 18\} \mathrm{kJ} . \text { The default of } \sigma_{b} \text { is } 5 \mathrm{~kJ} .\end{array}$ \\
\hline $\begin{array}{l}\text { Sensor working } \\
\text { time } w\end{array}$ & $\begin{array}{l}\text { Nine points in }[0.1,0.9] \text { with step time } 0.1 \mathrm{sec} \text { and } \\
\text { nine points in }[1,5] \text { with step time } 0.5 \mathrm{sec} \text {. The default } \\
\text { of } w \text { is } 1 \mathrm{sec} \text {. }\end{array}$ \\
\hline $\begin{array}{l}\text { Sensor density } \\
\qquad d\end{array}$ & $\begin{array}{l}d \sim N\left(\mu_{d}, \sigma_{d}\right) \text { where } \mu_{d}=\{2,4, \ldots, 20\} \text { and } \\
\sigma_{d}=\{0,1, \ldots, 6\} . \text { The default of }\left(\mu_{d}, \sigma_{d}\right) \text { is }(10,1) .\end{array}$ \\
\hline $\begin{array}{l}\text { Detection failure } \\
\text { probability } q\end{array}$ & $\begin{array}{l}q=\{0,0.05,0.1, \ldots, 0.1\} . \text { The default of } q \text { is } 0 \\
\text { that is, } 100 \% \text { detection. }\end{array}$ \\
\hline $\begin{array}{l}\text { Time } \\
\text { synchronization } \\
\text { error } \epsilon\end{array}$ & $\begin{array}{l}\epsilon \sim \text { Uniform }\left(-\epsilon_{\max }, \epsilon_{\max }\right) \text { where } \epsilon_{\max }=\{0,0.1 \text {, } \\
\ldots, 1\} \text { sec. The default of } \epsilon \text { is } 0 \text {, that is, no time } \\
\text { synchronization error. }\end{array}$ \\
\hline $\begin{array}{l}\text { Vehicle speed } \\
\qquad v\end{array}$ & $\begin{array}{l}v \sim N\left(\mu_{v}, \sigma_{v}\right) \text { where } \mu_{v}=\{15,20, \ldots, 60\} \mathrm{MPH} \\
\text { and } \sigma_{v}=\{0,1, \ldots, 10\} \mathrm{MPH} . \text { The maximum speed is } \\
70 \mathrm{MPH} \text { and the minimum speed } 10 \mathrm{MPH} . \text { The default } \\
\text { of }\left(\mu_{v}, \sigma_{v}\right) \text { is }(40,5) .\end{array}$ \\
\hline
\end{tabular}

For network lifetime measurement, the default energy budget $(50 \mathrm{~kJ})$ is used, but for the average detection time measurement, to obtain high statistical confidence, a full-day energy budget is used for the comparison among the three approaches.

\subsection{System Behavior over Time}

All three methods Virtual Scanning, Duty Cycling and AlwaysAwake can guarantee the detection of targets. Their difference lies in the network lifetime. Clearly, as a node can sleep longer per period with detection guarantee, the more energy efficiency can be obtained. Figure 15 shows how the sleeping time $T_{\text {sleep }}$ changes before network lifetime ends. As shown in the figure, Virtual Scanning has by far the longest sleeping time and hence the longest network lifetime. For example, Virtual Scanning sustains 
for 28.2 hours, compared with 1.4 hours in Duty Cycling and 5.4 minutes in Always-Awake. This is because of the significant energy savings during the scanning process. Note that in Figure 15, Virtual Scanning lets the sleeping time degrade gradually. This is because as the sensors are dying over time due to energy depletion, the sensing holes occur. These sensing holes let the distance between the entrance cluster and the protection cluster get shorter, as discussed in Section 3.3. Thus, the shorter intercluster distance leads to the shorter sleeping time. In the following subsections, we will quantitatively show the effect of the sleeping time on the performance.

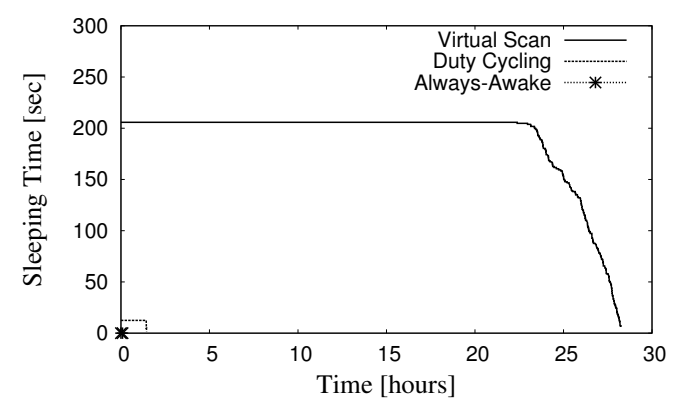

Fig. 15. The Comparison of Sleeping Time $T_{\text {sleep }}$ over Time

\subsection{Performance Comparison}

In this section, we compare three approaches: (i) Virtual Scanning, (ii) Duty Cycling and (iii) Always-Awake in terms of Network Lifetime and Average Detection Time under several user-level parameters, such as working time duration, energy budget, and sensor density. Each point in each experiment is the mean of the results obtained with 10 different random seeds.

\subsubsection{The Impact of Working Time}

Since $w$ is the minimum working time before reliable detection can be reported, this evaluation reveals how different hardware response speeds and sensing algorithms affect the VISA and other baselines. We use non-uniform $50 k J$ energy budget with the energy variation $5 k J$. Clearly, VISA provides significantly longer system lifetime than the baselines, especially when $w$ is large as shown in Figure 16(a). For example, when $w$ is 1 second, VISA extends network lifetime by 18.5 times, compared with Duty Cycling and 146 times, compared with Always-Awake. As shown Figure 16(b), the average detection time of Virtual Scanning is $11.5 \mathrm{sec}$, which is two times longer than that of Duty Cycling, 5.8 sec. Therefore, Virtual Scanning can provide 19 times lifetime of Duty Cycling at the expense of two times longer average detection time.

\subsubsection{The Impact of Sensor Density}

We define sensor density as the average number of sensors within sensing range $R$. As expected from the formula of the network lifetime in Eq. 2, the high sensor density provides the longer network lifetime for Virtual Scanning, as shown in Figure 17(a). This is because with a higher density, we have a longer scanning time $T_{\text {scan }}$, which allows sensor nodes to sleep longer. However, the high sensor density does not contribute much to the network lifetime to Duty Cycling and Always-Awake, since their sleeping time is independent of the number of sensors (as shown in Table 2).

For the average detection time, in both Virtual Scanning and Duty Cycling, under sparse sensor density less than 8 , the lower density lets the sensors close to entrances detect vehicles earlier, leading to the shorter average detection time, as shown in Figure 17(b). This is because for initial sensing holes due to low sensor density, the corresponding hole endpoints are labeled as entrance points or protection points for the sensing hole handling discussed in Section 3.3. As the sensor density becomes lower, the distance between the entrance cluster and the protection cluster is getting shorter, leading to the shorter average detection time according to Avg. Detection Time equations in Table 2. On the other hand, under sensor density greater than 8 , since the distance between the entrance cluster and the protection cluster is constant regardless of the sensor density, the average detection time is almost the same. In summary, at all sensor density settings, Virtual Scanning provides the longest network lifetime with a small degradation in detection time (e.g., double detection latency), compared with the lifetime increase (e.g., 19 times) where sensor density is 10 with $w=1 \mathrm{sec}$; note that this performance gain becomes higher when sensor density becomes higher.

\subsubsection{The Impact of Time Synchronization Error}

We investigate the impact of the time synchronization error on the network lifetime and average detection time. For this investigation, the margin of working time is set to the maximum time synchronization error $\epsilon_{\max }$ for the safe detection. As shown in Figure 18(a), as $\epsilon_{\max }$ increases, the network lifetimes of both Virtual Scanning and Duty Cycling decrease. This is because each sensor's working time increases by $2 \epsilon_{\max }$ and its sleeping time decreases by $2 \epsilon_{\max }$, as discussed in Section 3.5.

For the average detection time, as shown in Figure 18(b), Virtual Scanning has almost the same average detection time regardless of $\epsilon_{\max }$. This is because the average detection time is independent of the working time changed by $\epsilon_{\max }$, as shown in Table 2 . On the other hand, the average detection time of Duty Scanning tends to decrease as $\epsilon_{\max }$ increases. This is because the average detection time is inversely proportional to the working time increased by $2 \epsilon_{\max }$, as shown in Table 2.

\subsubsection{The Impact of Detection Failure Probability}

In this subsection, we investigate the impact of detection failure probability $q$ along with the following two parameters: (i) working time $w$ and (ii) sensor density $d$. Figure 19 shows the impact of the working time on the performance in the redundant sensing to deal with the detection failure probability. In the simulation setting, the number of redundant sensing activities is 3 because $q$ is set to 0.1 ; that is, since the working time in the redundant sensing is 3 times longer than that in the non-redundant sensing in Section 4.2.1, the lifetime in the redundant sensing is at most one third of that in the non-redundant sensing; note that this maximum lifetime is possible only when vehicles can be detected until the energy budgets of the sensors are depleted completely.

As shown in Figure 19(a), for Virtual Scanning, this expectation is valid when the working time is short, such as from 0.1 to $1 \mathrm{sec}$; this is caused by the fact that in a short working 


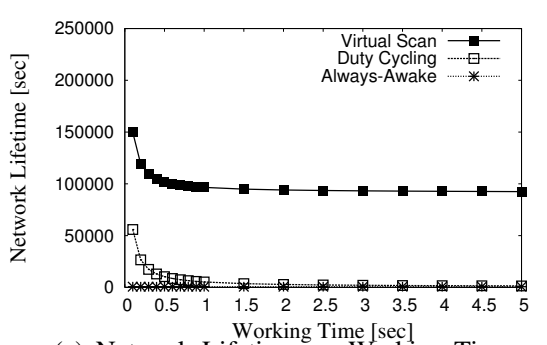

Working Time [sec]
(a) Network Lifetime vs. Working Time

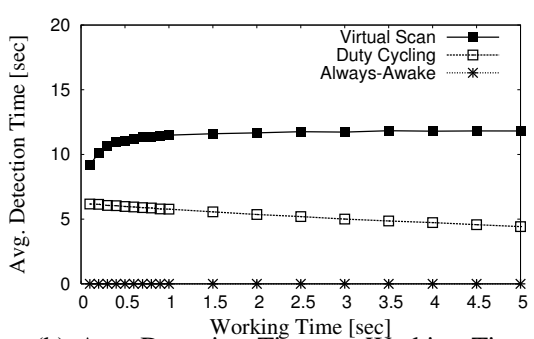

(b) Avg. Detection Time vs. Working Time

Fig. 16. The Impact of Working Time $w$

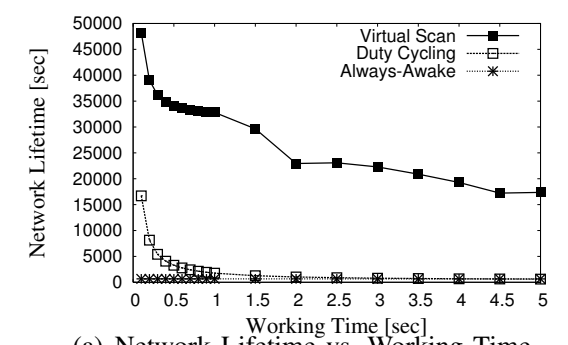

(a) Network Lifetime vs. Working Time

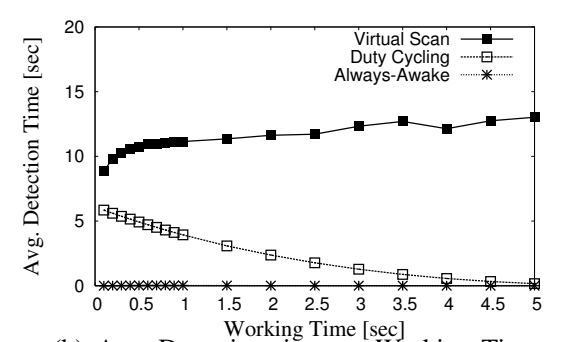

(b) Avg. Detection time vs. Working Time

Fig. 19. The Impact of Working Time $w$ under Redundant Sensing

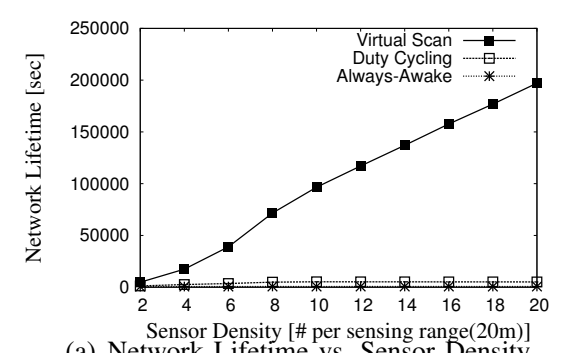

Sensor Density [\# per sensing range(20m)]
(a) Network Lifetime vs. Sensor Density

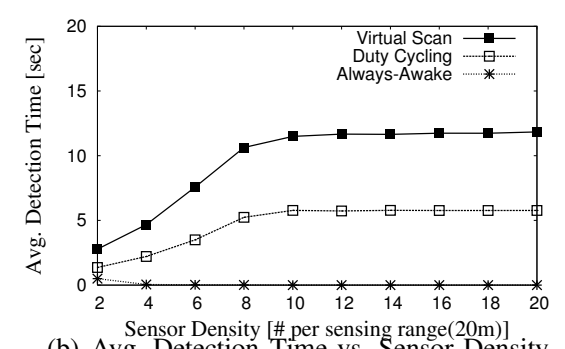

Sensor Density [\# per sensing range(20m)]
(b) Avg. Detection Time vs. Sensor Density

Fig. 17. The Impact of Sensor Density $d$

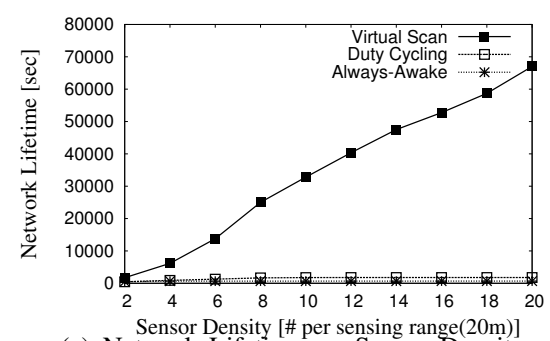

Sensor Density [\# per sensing range(20m)]
(a) Network Lifetime vs. Sensor Density

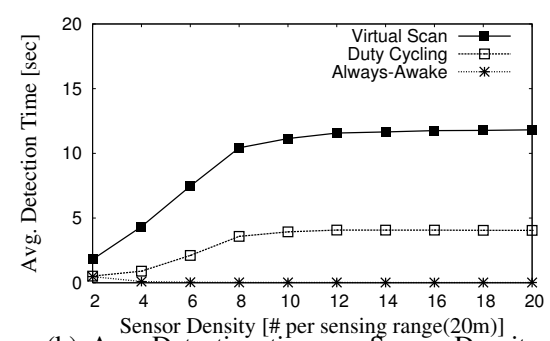

Sensor Density [\# per sensing range $(20 \mathrm{~m})]$
(b) Avg. Detection time vs. Sensor Density

Fig. 20. The Impact of Sensor Density $d$ under Redundant Sensing

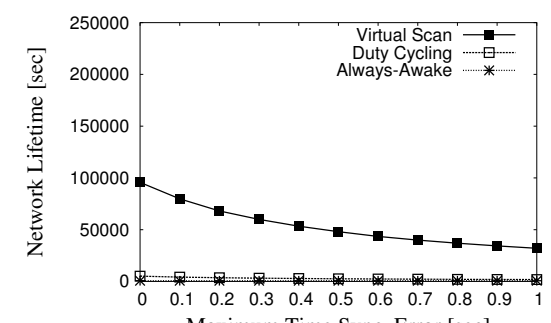

(a) Network Lifetime vs. Time Sync. Error

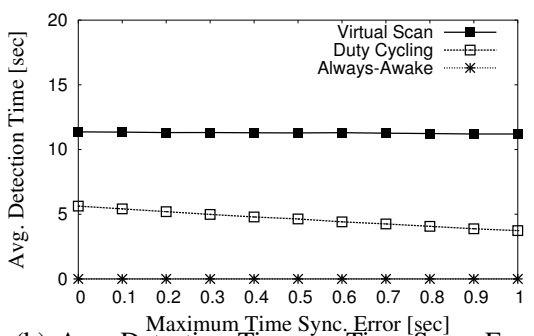

(b) Avg. Detection Time vs. Time Sync. Error

Fig. 18. The Impact of Time Synchronization Error $\epsilon_{\max }$

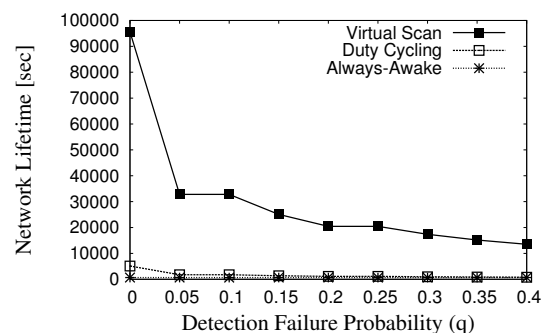

(a) Network Lifetime vs. Failure Probability

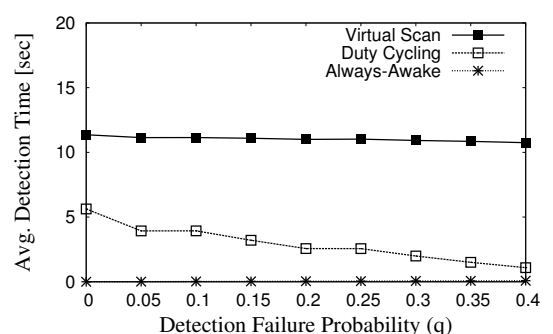

(b) Avg. Detection time vs. Failure Probability

Fig. 21. The Impact of Detection Failure Probability $q$

time, neighboring sensors can perform more than three sensing is not smooth from $w=1$ sec to $w=2$ sec. After $w=1$ activities. For example, suppose that two sensors $s_{1}$ and $s_{2}$ are sec, the network-wide detection failure probability dramatically almost in the same location and the working time $w$ is $0.1 \mathrm{sec}$. For increases with less spatial redundant sensing. For Duty Cycling, the sensing circle of 20 meters, a vehicle takes almost 1 second the lifetime tends to decrease as the working time increases in to pass this sensing circle with the speed of $40 \mathrm{MPH}$. These two the similar way with the case of the non-redundant sensing. For sensors can try to detect the vehicle 3 times, respectively; the total $w=0.1 \mathrm{sec}$, Virtual Scanning has 2.89 times longer lifetime than number of trials becomes 6. Thus, the network-wide detection Duty Cycling. For the working time longer than 0.5 sec, Virtual failure probability is $q^{6}=10^{-6}$ that is a very small number. On Scanning has at least 10 times longer lifetime than Duty Cycling. the other hand, for the working time longer than $1 \mathrm{sec}$, the lifetime As shown in Figure 19(b), the average detection time of Virtual is decreasing because the network-wide detection can fail earlier Scanning increases slightly as the working time increases. This is than the maximum lifetime with only three detection trials; in this because the network-wide detection failure probability increases case, the network-wide detection failure probability is $q^{3}=10^{-3}$. with less spatial redundant sensing according to the increase of the Thus, the benefit of the spatial redundant sensing decreases as working time, leading to a little later detection. For Duty Cycling, the working time increases. Note that in Figure 19(a), the curve the average detection time decreases faster for the increase of the 
working time than that in the non-redundant sensing, as shown in Figure 19(b). This is because the redundant sensing increases the working time per duty cycle; note that the average detection time is inversely proportional to the working time.

For the sensor density, as shown in Figure 20, the patterns of the curves of Virtual Scanning and Duty Cycling are similar to those in the non-redundant sensing, as shown in Figure 17. The remarkable difference in the network lifetime is that the lifetimes of Virtual Scanning and Duty Cycling are reduced to almost one third. For the average detection time, Virtual Scanning has almost the same detection time as with the case of the non-redundant sensing. The average detection time of Duty Cycling is shorter than the case of the non-redundant sensing. This is because that the average detection time is inversely proportional to the working time and the working time is increased due to the redundant sensing.

Now, we investigate the impact of detection failure probability on both peformance metrics. For the lifetime, as shown in Figure 21(a), Virtual Scanning has shorter lifetime according to the increase of detection failure probability $q$, leading to the increasing number of sensing activities per duty cycle. However, the performance gain of Virtual Scanning in the lifetime is at least 17 times over Duty Cycling and at least 20 times over AlwaysAwake. For the average detection time, as shown in Figure 21(b), Virtual Scanning has almost the constant detection time, however Duty Cycling tends to have a shorter detection time according to the increase of $q$; this is because the increase of $q$ leads to the increase of the working time.

In summary, even in the realistic setting with the time synchronization error and detection failure probability, Virtual Scanning outperforms both Duty Cycling and Always-Awake in terms of network lifetime.

\subsection{Achieving Shorter Delay and Longer Lifetime Simul- taneously}

In this subsection, we show that there exists a working time such that Virtual Scanning is better in both the detection delay and the lifetime than Duty Cycling. In Section 2.4, we showed analytically how VISA achieves a shorter delay and a longer network lifetime simultaneously by adjusting the silent time $\left(T_{\text {silent }}=\alpha\right)$ within the range that satisfies Eq. 3 where the silent time is part of the sleeping time. To confirm our design empirically, Figure 22 shows the performance effect of Virtual Scanning according to $\alpha$. As shown in Figure 22, when Virtual Scanning reduces $\alpha$ from $T_{\text {silent }}$ to 0 in the working time of 0.1 second, it has better performance in both the network lifetime and average detection time than Duty Cycling. Therefore, the system operator can achieve the required performance by tuning the working time and the sleeping time.

\subsection{The Effect of Hole Handling}

This section compares three different methods for hole handling:

- MST-based Labeling: our hole labeling scheme discussed in Section 3.3.

- Random Labeling: a new hole is randomly labeled as either pseudo entrance point or pseudo protection point.

- No Labeling: when a new hole occurs, it is not handled, leading to the end of system lifetime.

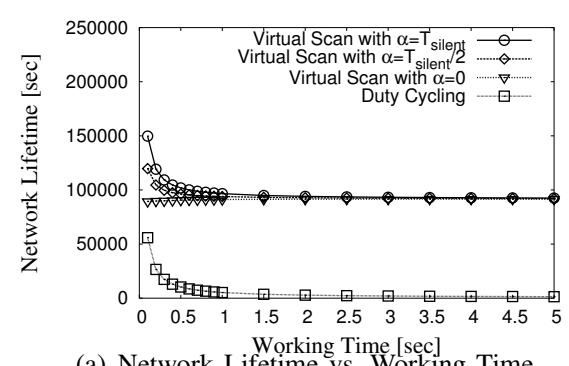

Working Time $[\mathrm{sec}]$
(a) Network Lifetime vs. Working Time

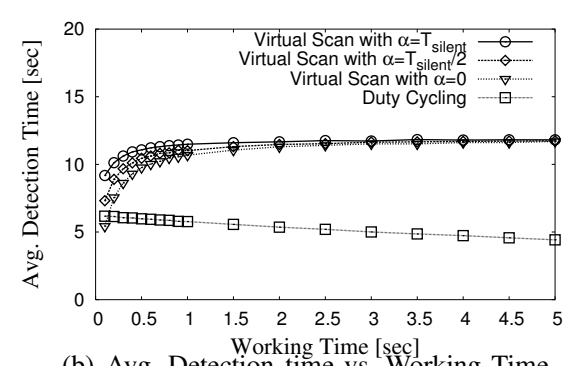

(b) Avg. Detection time vs. Working Time

Fig. 22. The Impact of Silent Time $\alpha$

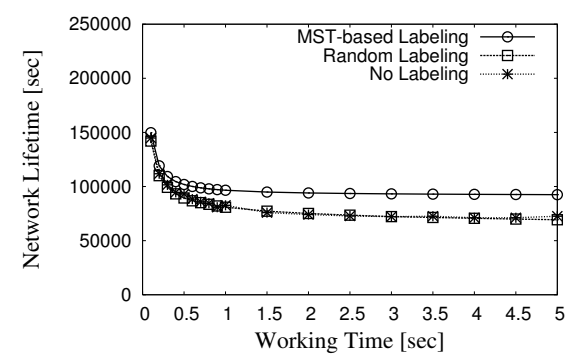

Fig. 23. The Comparison of Hole Labeling Algorithms

We use the same Virtual Scanning for these three labeling algorithms. As shown in Figure 23, MST-based Labeling gives longer lifetime than both Random Labeling and No Labeling. Random Labeling and No Labeling have the similar lifetime, because Random Labeling cannot label holes appropriately to prevent a breach path (i.e., path vulnerable to vehicle intrusion to protection points) from existing; that is, since Random Labeling might label holes against our labeling rule based on MST discussed in Section 3.3.1 (i.e., to label two closest holes with the same label for longer lifetime), the holes close to entrance points can have different labels, leading to the short sleeping time. Since No Labeling does not handle sensing hole, one sensing hole creates a breach path, leading to the end of system.

For the average detection time, these three labeling algorithms have similar performance whose curves are almost the same as the curve of Virtual Scanning in Figure 16(b).

\section{Related Work}

Most research on coverage for detection has so far focused on Full Coverage [1]-[4], [17]-[20] in a 2-dimensional space. In [4], authors use the off-duty eligibility rule to turn on/off a node as long as the neighboring nodes can cover the sensing area of this node. The Coverage Configuration Protocol (CCP) [18] provides an energy-efficient sensing coverage, integrated with SPAN for connectivity. In [21], surveillance coverage is achieved through probing. DiffSurv [22] provides differentiated surveillance to an 
area with a certain degree of coverage, up to the limitation imposed by the number of sensor nodes deployed. Compared with this static coverage, Cheng et al. [23] propose sweep coverage based on mobile sensors. These mobile sensors monitor certain points of interest such that these points are swept by the mobile sensors at least once every required period. Cheng et al. also investigate theoretically the minimum number of mobile sensors to satisfy the required sweep coverage. Kumar et al. [3] identify a critical bound for k-coverage in a network, assuming a node is randomly turned on with a certain probability. In [2], Cardei et al. propose two heuristic algorithms to identify a maximum number of set covers to monitor a set of static targets at known locations. In [1], Abrams et al. propose three approximation algorithms for a relaxed version of the previously defined SET K-COVER problem [24]. Even for SET K-COVER setting, our Virtual Scanning outperforms the Duty Cycling in terms of lifetime. For each cover, Virtual Scanning has a longer schedule period according to scan time than Duty Cycling, leading to the longer lifetime. We leave the application of SET-K-COVER to Virtual Scanning as future work.

To aggressively reduce energy consumption, partial coverage through Duty Cycling has been studied as well. In [25], [26], authors provide a theoretical analysis and simulation on the delay (or stealth distance) before a target is detected. In [25], the Quality of Surveillance (QoSv) is defined as the reciprocal value of the expected travel distance before mobile targets are first detected by any sensor. In [27], nodes coordinate among each other to guarantee the worst-case detection delay and minimize the average detection delay. In [28]-[30], the theoretical foundations for laying barriers with stealthy and wireless sensors are proposed in order to detect the intrusion of mobile targets approaching the barriers from the outside.

The closest related work is virtual patrol [31], in which a virtual patrol moves along the predefined path in 2-dimensional space and triggers sensors adjacent to the virtual patrol's path for detection. This virtual patrol is similar to the concept of our virtual scan. However, the uniqueness of our work can be clearly identified from the following respects: (i) our work focuses on surveillance in road network, where legacy 2-dimensional solutions cannot directly apply, and (ii) we are the first to guarantee target detection with sensing hole handling while sensor network deteriorates.

\section{Conclusion}

Specially tailored for road networks, this work introduces VISA based on the concept of virtual scanning. VISA propagates sensing waves along the roadways and detects vehicles entering into the target road network before they reach the protection points. We demonstrate analytically and empirically the feasibility of achieving longer network lifetime and shorter detection delay simultaneously. In addition, we propose an optimal algorithm to deal with the initial sensing holes at the deployment time as well as the sensing holes due to node failure and the heterogeneous energy budget among sensors by optimally labeling additional pseudo protection or entrance points. Evaluation shows orders-ofmagnitude longer network lifetime than the always-awake method, and as much as ten times longer than the duty cycling algorithms. We believe this work opens a promising direction of road network surveillance. Future work includes (i) the perimeter protection of road networks, (ii) protection design with bounded detection delay for the quality of surveillance, (iii) optimal sensor placement with minimal detection delay, and (iv) civil applications, such as the contamination detection in water flow systems.

\section{ACKNOWLEDGMENT}

This research was supported by the Digital Technology Center at the University of Minnesota, and in part by NSF grants CNS0626609, CNS-0626614, and CNS-0720465.

\section{References}

[1] Z. Abrams, A. Goel, and S. Plotkin, "Set K-Cover Algorithms for Energy Efficient Monitoring in Wireless Sensor Networks," in IPSN. ACM/IEEE, 2004.

[2] M. Cardei, M. T. Thai, Y. Li, and W. Wu, "Energy-Efficient Target Coverage in Wireless Sensor Networks," in IEEE. INFOCOM, 2005.

[3] S. Kumar, T. H. Lai, and J. Balogh., "On K-Coverage in a Mostly Sleeping Sensor Network," in MOBICOM. ACM, 2004.

[4] D. Tian and N. Georganas, "A Node Scheduling Scheme for Energy Conservation in Large Wireless Sensor Networks," Wireless Communications and Mobile Computing Journal, May 2003.

[5] A. Savvides, C. C. Han, and M. B. Srivastava, "Dynamic Fine-Grained Localization in Ad-Hoc Networks of Sensors," in MOBICOM. Rome, Italy: ACM, Jul. 2001.

[6] J. Elson, L. Girod, and D. Estrin, "Fine-Grained Network Time Synchronization using Reference Broadcasts," in OSDI. ACM, Dec. 2002.

[7] M. Maróti, B. Kusy, G. Simon, and Ákos Lédeczi, "The Flooding Time Synchronization Protocol," in SENSYS. Baltimore, Maryland, USA: ACM, Nov. 2004.

[8] Lin Gu et al., "Lightweight Detection and Classification for Wireless Sensor Networks in Realistic Environments," in SENSYS. San Diego, California, USA: ACM, Nov. 2005

[9] J. Hwang, T. He, and Y. Kim, "Exploring In-Situ Sensing Irregularity in Wireless Sensor Networks," in SENSYS. ACM, Nov. 2007, pp. 289-303.

[10] Y. Gu and T. He, "Data Forwarding in Extremely Low Duty-Cycle Sensor Networks with Unreliable Communication Links," in SENSYS. Sydney, Australia: ACM, Nov. 2007, pp. 321-334.

[11] G. Lu, N. Sadagopan, B. Krishnamachari, and A. Goel, "Delay Efficient Sleep Scheduling in Wireless Sensor Networks," in INFOCOM. IEEE, 2005.

[12] T. H. Cormen, C. E. Leiserson, R. L. Rivest, and C. Stein, Introduction to Algorithms (2nd Edition). MIT Press, 2003.

[13] Q. Fang, J. Gao, and L. J. Guibas, "Locating and Bypassing Routing Holes in Sensor Networks," in INFOCOM. IEEE, Mar. 2004.

[14] S. Funke and C. Klein, "Hole Detection or: "How much Geometry hides in Connectivity?"," in SoCG. ACM, Jun. 2006.

[15] T. Asano, B. Bhattacharya, M. Keil, and F. Yao, "Clustering Algorithms based on Minimum and Maximum Spanning Trees," in Proceedings of the Fourth Annual Symposium on Computational Geometry, 1988.

[16] Tian He et al., "An Energy-Efficient Surveillance System Using Wireless Sensor Networks," in MOBISYS. ACM, Jun. 2004.

[17] S. Meguerdichian, F. Koushanfar, M. Potkonjak, and M. B. Srivastava, "Coverage Problems in Wireless Ad-hoc Sensor Networks," in INFOCOM. Anchorage, Alaska: IEEE, Apr. 2001.

[18] X. Wang, G. Xing, Y. Zhang, C. Lu, R. Pless, and C. Gill, "Integrated Coverage and Connectivity Configuration in Wireless Sensor Networks," in SENSYS. Los Angeles, CA, USA: ACM, Nov. 2003.

[19] S. Shakkottai, R. Srikant, and N. Shroff, "Unreliable Sensor Grids: Coverage, Connectivity, and Diameter," in INFOCOM. San Francisco, CA, USA: IEEE, Apr. 2003.

[20] H. Zhang and J. Hou, "Maintaining Sensing Coverage and Connectivity in Large Sensor Networks," Ad Hoc \& Sensor Wireless Networks, vol. 1, Mar. 2005.

[21] F. Ye, G. Zhong, J. Cheng, S. Lu, and L. Zhang, "PEAS: A Robust Energy Conserving Protocol for Long-lived Sensor Networks," in ICDCS. IEEE, May 2003.

[22] T. Yan, T. He, and J. Stankovic, "Differentiated Surveillance Service for Sensor Networks," in SENSYS. Los Angeles, CA, USA: ACM, Nov. 2003.

[23] W. Cheng, M. Li, K. Liu, Y. Liu, X. Li, and X. Liao, “"Sweep Coverage with Mobile Sensors"," in IPDPS. IEEE, Apr. 2008.

[24] S. Slijepcevic and M. Potkonjak, "Power Efficient Organization of Wireless Sensor Networks," in ICC. IEEE, 2001. 
[25] C. Gui and P. Mohapatra, "Power Conservation and Quality of Surveillance in Target Tracking Sensor Networks," in MOBICOM. Philadelphia, PA, USA: ACM, Sep. 2004.

[26] S. Ren, Q. Li, H. Wang, X. Chen, and X. Zhang, "Analyzing Object Tracking Quality under Probabilistic Coverage in Sensor Networks," ACM Mobile Computing and Communications Review, vol. 9, no. 1, Jan. 2005.

[27] Q. Cao, T. Abdelzaher, T. He, and J. Stankovic, "Towards Optimal Sleep Scheduling in Sensor Networks for Rare Event Detection ", in IPSN. ACM/IEEE, 2005.

[28] S. Kumar, T. Lai, and A. Arora, "Barrier Coverage With Wireless Sensors," in MOBICOM. Cologne, Germany: ACM, Aug. 2005.

[29] A. Chen, S. Kumar, and T.-H. Lai, "Designing Localized Algorithms for Barrier Coverage," in MOBICOM. ACM, Sep. 2007.

[30] P. Balister, B. Bollobás, A. Sarkar, and S. Kumar, "Reliable Density Estimates for Achieving Coverage and Connectivity in Thin Strips of Finite Length ," in MOBICOM. ACM, Sep. 2007.

[31] C. Gui and P. Mohapatra, "Virtual Patrol: A New Power Conservation Design for Surveillance Using Sensor Networks," in IPSN. ACM/IEEE, Apr. 2005

\section{APPENDIX A \\ Average Detection time in Virtual Scan- NING}

In this section, we derive the Average Detection Time (ADT) for virtual scanning in a road segment. At first, for clarity, we assume vehicle speed is constant, the same as with the maximum speed $v$. Later, we relax this assumption; that is, vehicle speed is bounded variable speed.

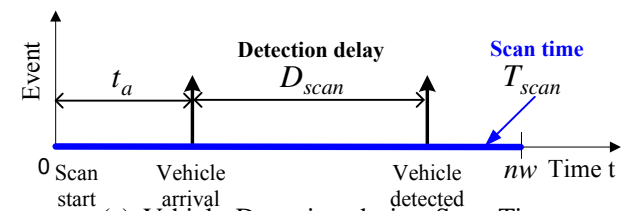

(a) Vehicle Detection during Scan Time

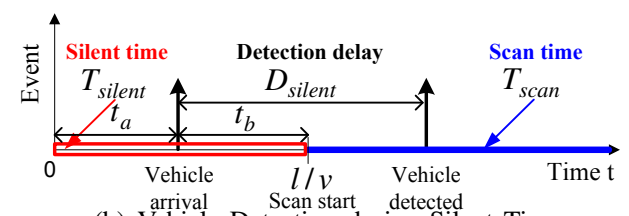

(b) Vehicle Detection during detected

Fig. 24. Vehicle Detection Cases in Virtual Scanning

Enter during Scan Time: Figure 24(a) shows a vehicle enters during the scan time $T_{\text {scan }}$. Since each node covers road segment of length $l / n$, the virtual scan wave moves along the road segment with the speed $v_{\text {scan }}=l /(n w)$. The relative speed between the scan wave and the vehicle is $l /(n w)+v$. Suppose a vehicle enters at $t_{a}$ after the start of scan, the scan wave has already traveled $l t_{a} /(n w)$. Therefore it takes $\left(l-\frac{l t_{a}}{n w}\right) /\left(\frac{l}{n w}+v\right)$ seconds before the scan wave reaches the vehicle, which is the detection delay $D_{\text {scan }}$. Integrated $t_{a}$ over the interval $[0, n w]$, expected detection delay (denoted as $E\left[D_{\text {scan }}\right]$ ) during scan time is:

$$
E\left[D_{\text {scan }}\right]=\int_{0}^{n w} \frac{n w l-l t_{a}}{n w v+l} \frac{1}{n w} d t_{a}=\frac{n w l}{2(n w v+l)} .
$$

Enter during Silent Time: Figure 24(b) shows a vehicle enters during the silent time $T_{\text {silent }}$. Suppose a vehicle enters at $t_{a}$ after the start of silent time. As shown in Figure 24(b), since it enters at $t_{b}$ before the start of scan, the vehicle has already traveled $t_{b} v$. Therefore it takes $\left(l-t_{b} v\right) /\left(\frac{l}{n w}+v\right)$ seconds before the scan wave reaches the vehicle. For the detection delay, we also need to count the vehicle movement time $t_{b}$ along with the previous detection delay after the start of the scan. Note that $t_{b}=l / v-t_{a}$. Thus the detection delay becomes $D_{\text {silent }}=\frac{l}{v}-t_{a}+\left(l-\left(\frac{l}{v}-t_{a}\right) v\right) /\left(\frac{l}{n w}+\right.$ $v)$. Integral $t_{a}$ over the interval $[0, l / v]$, expected detection delay (denoted as $E\left[D_{\text {silent }}\right]$ ) during the silent time is:

$$
E\left[D_{\text {silent }}\right]=\int_{0}^{l / v} \frac{n w l-l t_{a}+l^{2} / v}{n w v+l} \frac{v}{l} d t_{a}=\frac{2 n w l+l^{2} / v}{2(n w v+l)} .
$$

Combined both scenarios, we can compute the expected ADT for the virtual scanning as follows:

$$
E[D]=\frac{n w}{n w+l / v} E\left[D_{\text {scan }}\right]+\frac{l / v}{n w+l / v} E\left[D_{\text {silent }}\right]=\frac{l}{2 v} .
$$

\section{APPENDIX B \\ ADT COMPUTATION FOR BOUNDED VARIABLE Vehicle SPEED}

Now we relax the assumption that vehicle speed is constant, the same as with the maximum speed $v_{\max }$. We assume that vehicle speed is bounded variable speed $v=\left[v_{\min }, v_{\max }\right]$ for $0<v_{\min }<$ $v_{\max }$. Since this relaxation causes the silent time to be changed as $T_{\text {silent }}=l / v_{\max }$, the expected detection delay during the silent time becomes as follows:

$$
\begin{aligned}
E\left[D_{\text {silent }}\right] & =\int_{0}^{l / v_{\max }} \frac{n w l-l t_{a}+l^{2} / v_{\max }}{n w v+l} \frac{v_{\max }}{l} d t_{a} \\
& =\frac{2 n w l+l^{2} / v_{\max }}{2(n w v+l)} .
\end{aligned}
$$

Since there exists no change in the detection delay during the scan time, the combined expected ADT is:

$$
\begin{aligned}
E[D] & =\frac{n w}{n w+l / v_{\max }} E\left[D_{\text {scan }}\right]+\frac{l / v_{\max }}{n w+l / v_{\max }} E\left[D_{\text {silent }}\right] \\
& =\frac{l\left(n w v_{\max }+l\right)}{2 v_{\max }(n w v+l)} .
\end{aligned}
$$

Clearly, Eq. 10 becomes the same one as with Eq. 8 for $v=v_{\max }$.

Now we can compute the average detection time for bounded variable vehicle speed. Suppose that the vehicle speed has a Gaussian distribution with $N\left(\mu_{v}, \sigma_{v}\right)$ in the range of $v=\left[v_{\min }, v_{\max }\right]$. We can compute the expected ADT for this setting as follows:

$$
E[D]=\int_{v_{\min }}^{v_{\max }} \frac{l\left(n w v_{\max }+l\right)}{2 v_{\max }(n w v+l)} \frac{1}{\sqrt{2 \pi} \sigma_{v}} e^{-\frac{\left(v-\mu_{v}\right)^{2}}{2 \sigma_{v}^{2}}} d v .
$$

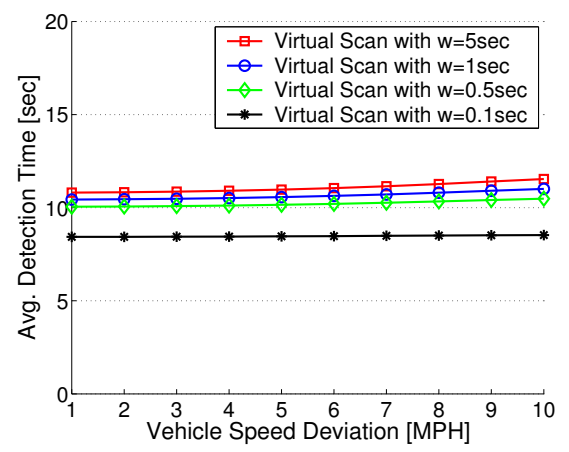

Fig. 25. The Impact of Vehicle Speed Deviation on Average Detection Time

As shown in Figure 25, we can see the trend of ADT according to the vehicle speed deviation in bounded variable speed, where $v_{\max }=70 \mathrm{MPH}, v_{\min }=10 \mathrm{MPH}, \mu_{v}=40 \mathrm{MPH}$, and $\sigma_{v}=\{1,2, \ldots, 10\} \mathrm{MPH}$. It can be observed that the ADT tends to slightly increase as the vehicle speed deviation $\sigma_{v}$ increases. 


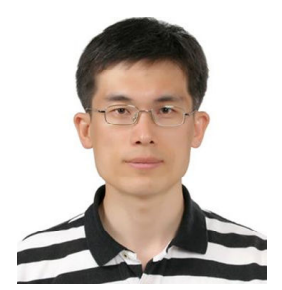

Jaehoon Jeong is a Ph.D. candidate in the Department of Computer Science and Engineering at the University of Minnesota. He received the B.S. degree from the department of Information Engineering at Sungkyunkwan University in Korea in 1996 and the M.S. degree in the School of Computer Science and Engineering at Seoul National University in Korea in 2001. Also, as a researcher in Electronics and Telecommunications Research Institute (ETRI), he has participated in the Internet Standardization in the Internet Engineering Task Force (IETF), such as IPv6 DNS Configuration. He has published two IETF standards of RFC 4339 and RFC 5006 for IPv6 DNS Configuration. His current research interests are the wireless sensor networking for road networks and the data forwarding in vehicular networks. He is a member of the IEEE and the ACM.

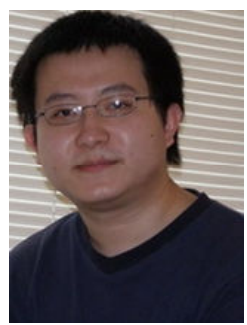

Yu Gu is currently a Ph.D candidate in the Department of Computer Science at the University of Minnesota. His research interests include wireless sensor networks, real-time computing and distributed systems. He is the author and co-author of over 17 papers in premier journals and conferences. His publications have been selected as graduate-level course materials by over 10 universities worldwide. $\mathrm{He}$ is a member of ACM, IEEE and SIAM.

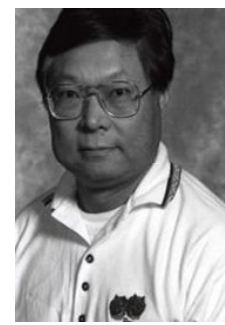

Dr. David H.C. Du is currently a Qwest Chair Professor of Computer Science and Engineering at University of Minnesota, Minneapolis. He is the Center Director of a new established NSF multi-university I/UCRC Center on Intelligent Storage (joint with UC Santa Cruz). He was a Program Director (IPA) at National Science Foundation CISE/CNS Division from March 2006 to August 2008. At NSF, he was responsible for NeTS (Networking Research cluster) NOSS (Networks of Sensor Systems) Program and worked with two other colleagues, Karl Levitt and Ralph Wachter, on Cyber Trust (Internet Security) Program. In 2008 he was also assigned to CSR (Computer System Research) Cluster. Dr. Du receives a B.S degree in Mathematics from National Tsing-Hua University (Taiwan) in 1974 and an M.S. and Ph.D. degrees from University of Washington (Seattle) in 1980 and 1981 respectively. He joined University of Minnesota as a faculty since 1981. Dr. David Du's current research focuses on intelligent storage systems, multimedia computing, sensor networks and cyber physical systems. He has had research in high-speed networking, database design, and CAD for VLSI circuits. He has authored and co-authored over 200 technical papers including 95 referred journal publications in these research areas. He has graduated 49 Ph.D. and 80 M.S. students in the last 25 years. His research in sensor networks and cyber physical systems focuses on vehicle-to-vehicle communications, future intelligent transportation systems, next generation of air-transportation systems and security and privacy issues for cyber physical systems. Dr. Du is an IEEE Fellow (since 1998) and a Fellow of the Minnesota Supercomputer Institute. He is currently a member of Advisory Committee to Institute of Information Science, Academic Sinica and a member of International Advisory Board to Korean Journal of Computer Science and Engineering.

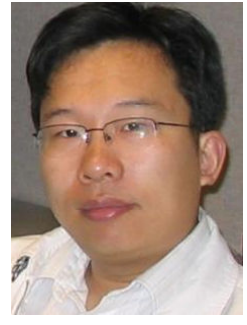

Dr. Tian He is currently an assistant professor in the Department of Computer Science and Engineering at the University of Minnesota-Twin City. He received the Ph.D. degree under Professor John A. Stankovic from the University of Virginia, Virginia in 2004. Dr. $\mathrm{He}$ is the author and co-author of over 90 papers in premier sensor network journals and conferences with over 4000 citations. His publications have been selected as graduate-level course materials by over 50 universities in the United States and other countries. Dr. He has received a number of research awards in the area of sensor networking, including four best paper awards (MSN 2006 and SASN 2006, MASS 2008, MDM 2009). Dr. He is also the recipient of the NSF CAREER Award 2009 and McKnight Land-Grant Professorship 2009-2011. Dr. He served a few program chair positions in international conferences and on many program committees, and also currently serves as an editorial board member for four international journals including ACM Transactions on Sensor Networks. His research includes wireless sensor networks, intelligent transportation systems, real-time embedded systems and distributed systems, supported by National Science Foundation and other agencies. Dr. He is a member of ACM and IEEE. 